Continuum and Emission Line Strength Relations for a large Active Galactic Nuclei Sample

Dietrich, M.; Hamann, F.; Shields, J. C.; Constantin, A.; Vestergaard, Marianne; Chaffee, F.; Foltz, C. B.; Junkkarinen, V. T.

Published in:

Astrophysical Journal

DOI:

$10.1086 / 344410$

Publication date:

2002

Citation for published version (APA):

Dietrich, M., Hamann, F., Shields, J. C., Constantin, A., Vestergaard, M., Chaffee, F., Foltz, C. B., \&

Junkkarinen, V. T. (2002). Continuum and Emission Line Strength Relations for a large Active Galactic Nuclei Sample. Astrophysical Journal, 581(2), [912]. https://doi.org/10.1086/344410 
To be published in Astrophysical Journal

\title{
Continuum and Emission Line Strength Relations for a large Active Galactic Nuclei Sample
}

\author{
M. Dietrich ${ }^{1}$ and F. Hamann ${ }^{1}$ \\ Department of Astronomy, University of Florida, 211 Bryant Space Science Center, \\ Gainesville, FL 32611-2055, USA \\ J.C. Shields ${ }^{2}$ and A. Constantin ${ }^{2}$ \\ Department of Physics 83 Astronomy, Ohio University, Clippinger Research Labs.251B, \\ Athens, $\mathrm{OH}$ 45701-2979, USA \\ M. Vestergaard ${ }^{3}$ \\ Department of Astronomy, The Ohio State University, 140 West 18th Av., Columbus, OH \\ 43210-1173, USA. \\ F. Chaffee ${ }^{4}$ \\ W.M. Keck Observatory, 65-1120 Mamalahoa Hwy., Kamuela, HI 96743, USA \\ C.B. Foltz ${ }^{5}$ \\ Multiple Mirror Telescope Observatory, University of Arizona, Tucson, AZ 85721, USA \\ and \\ V.T. Junkkarinen ${ }^{6}$ \\ Center for Astrophysics and Space Sciences, Code 0424, 9500 Gilman Drive, University of \\ California at San Diego, La Jolla, CA 92093-0424, USA \\ dietrich@astro.ufl.edu
}

\begin{abstract}
We report on the analysis of a large sample of 744 type 1 Active Galactic Nuclei, including quasars and Seyfert 1 galaxies across the redshift range from $0 \lesssim z \lesssim 5$ and spanning nearly 6 orders of magnitude in continuum luminosity.
\end{abstract}


We discuss correlations of continuum and emission line properties in the restframe ultraviolet and optical spectral ranges. The well established Baldwin Effect is detected for almost all emission lines from O vi $\lambda 1034$ to [O III] $\lambda 5007$. Their equivalent widths are significantly anti-correlated with the continuum strength, while they are nearly independent of redshift. This is the well known Baldwin Effect. Its slope $\beta$, measured as $\log W_{\lambda} \propto \beta \log \lambda L_{\lambda}(1450 \AA)$, shows a tendency to become steeper towards higher luminosity. The slope of the Baldwin Effect also increases with the ionization energy needed to create the individual lines. In contrast to this general trend, the $\mathrm{N} v \lambda 1240$ equivalent width is nearly independent of continuum luminosity and remains nearly constant. The overall line behaviors are consistent with softer UV continuum shapes and perhaps increasing gas metallicity in more luminous Active Galactic Nuclei.

Subject headings: quasars: emission lines, galaxies: active

\section{Introduction}

Broad emission lines (BELs) are a defining property of quasar spectra. Nearly 25 years ago Baldwin (1977) discovered an anti-correlation between the equivalent width in C IV $\lambda 1549$, $W_{\lambda}(\mathrm{C}$ IV $)$, and the continuum luminosity, $L_{\lambda}(1450 \AA)$, measured at $1450 \AA$ in the quasar restframe. This result was based on a sample of 20 quasars spanning $\sim 2$ orders of magnitude in continuum luminosity in the redshift range $1.24 \lesssim z \lesssim 3.53$. It has become known as the "Baldwin Effect" (hereafter BEff). The BEff was subsequently confirmed in C IV $\lambda 1549$, as well as other BELs such as Ly $\alpha$ and O vi $\lambda 1034$ (Véron-Cetty, Véron, \& Tarenghi 1983; Baldwin, Wampler, \& Gaskell 1989; Kinney, Rivolo, \& Koratkar 1990; Osmer, Porter, \& Green 1994; Zheng, Kriss, \& Davidsen 1995; Green, Forster, \& Kuraszkiewicz 2001; see Osmer \& Shields 1999 for a recent review).

A major impetus for studying the BEff was that it might be useful for calibrating Active Galactic Nuclei (AGNs) luminosities, e.g. based on $W_{\lambda}(\mathrm{CIV})$. The AGNs could then be used as cosmological standard candles. But in the following years studies of bigger quasar samples revealed a large scatter in the anti-correlation of the continuum luminosity vs. emission line strength (Baldwin, Wampler, \& Gaskell 1989; Zamorani et al. 1992). The BEff is nonetheless important as a diagnostic of AGN structure and, perhaps, metal abundances (Korista, Baldwin, \& Ferland 1998). The relation between the continuum luminosity and the relative emission line strengths and ratios can be used to study the evolution and physics of the quasar phenomenon (Baldwin 1999). In particular, this correlation can be used to test model predictions for the dependence of the shape of the continuum spectral energy 
distribution as a function of luminosity (Binette et al. 1989; Netzer, Laor, \& Gondhalekar 1992; Zheng \& Malkan 1993; Wandel 1999a,b).

The most fundamental problem, however, is that the physical cause of the BEff remains unknown. It was suggested by Mushotzky \& Ferland (1984) that the observed relation can be explained by an anti-correlation of the ionization parameter, $\mathrm{U}$, and the continuum luminosity, with $U=Q(H) / 4 \pi r^{2} c n_{H}$, where $\mathrm{Q}(\mathrm{H})$ is the number of hydrogen ionizing photons emitted per second by the central continuum source, $\mathrm{r}$ is the distance between the continuum source and the emission line region, and $n_{H}$ is the hydrogen density in the line-emitting cloud. Assuming an additional relation of decreasing covering factor with increasing continuum strength, the observed BEff could be well described for C IV $\lambda 1549$. However, this model does not naturally explain the BEff in the full range of measured lines. It predicts, for example, a lack of a BEff for Ly $\alpha$ even though it is clearly detected (e.g., Kinney, Rivolo, \& Koratkar 1990; Osmer, Porter, \& Green 1994; Laor et al. 1995; Green 1996).

Another important clue to the physical cause of the BEff is that the strength of the relationship (i.e., the slope of the $W_{\lambda}-L_{c}$ anti-correlation) seems to depend on the ionization energy of the emission-line species (Zheng et al.1995; Espey \& Andreadis 1999). In particular, the equivalent widths of high-ionization lines like O vi $\lambda 1034$ decrease more dramatically with $L_{c}$ than lines with moderate ionization energies like C IV $\lambda 1549$ or low ionization energies like Mg II $\lambda 2798$ and Balmer emission lines. Our results in the present paper confirm this claim, and improve upon the overall empirical characterization of the BEff correlations.

The BEff might result from a more fundamental correlation between the continuum luminosity, $L_{c}$, and the shape of the ionizing (EUV-soft X-ray) continuum (Binette et al. 1989; Zheng \& Malkan 1993; Zheng et al. 1997; Korista et al. 1998). Netzer (1985, 1987) and Netzer et al. (1992) suggested accretion disc models to explain the observed continuum and emission line correlations. Recently, Wandel $(1999 \mathrm{a}, \mathrm{b})$ added to this the growth in black hole mass by accretion in the accretion disc model. His analysis predicts that the continuum luminosity increases towards higher black hole mass and that the shape of the ionizing continuum becomes softer. Hence, it is suggested that the BEff is driven by a softening of the ionizing continuum towards higher luminosities. This model is attractive because the UV - X-ray spectral softening has been well documented by observations (Tananbaum et al. 1986; Wilkes et al.1994; Green et al. 1995), and because the spectral softening provides a natural explanation for steeper BEff slopes in higher ionization lines.

An additional possibility is that the BEff is driven, at least in part, by a trend for higher metallicities in more luminous AGNs. Korista et al. (1998) presented theoretical results showing the dependence of the emission line equivalent widths on both the continuum shape and the metallicity of the gas. Their proposal is based on evidence for higher metal 
abundances in more luminous quasars (Hamann \& Ferland 1993, 1999; Dietrich et al. 1999; Dietrich \& Wilhelm-Erkens 2000), and on the suggestion that more luminous quasars reside in more massive host galaxies, which will naturally be more metal rich (Cen \& Ostriker 1999; Pettini 1999; Kauffmann \& Haehnelt 2000; Granato et al. 2001; see Hamann \& Ferland 1999 for a review).

Our combination of ground-based and satellite data also provides the opportunity to study evolutionary aspects of the line strengths in detail — e.g. by measuring the same rest-frame UV lines across a wide range of redshifts. One serious complication affecting previous work is that luminosity and redshift are often correlated in quasars samples, because the quasars are selected from magnitude-limited surveys. Most studies have supported the original claim by Baldwin (1977), that $\mathrm{W}_{\lambda}$ scales inversely with luminosity and that there is no significant trend with redshift $z$ (e.g., Kinney, Rivolo, \& Koratkar 1990; Osmer et al. 1994; Francis \& Koratkar 1995; Wilkes et al. 1999). However, some recent work based on the Large Bright Quasar Survey (LBQS) claims that the relationship to redshift is even stronger than with luminosity (Forster et al. 2001; Green, Forster, \& Kuraszkiewicz 2001).

The present paper is the first in a series in which we examine the emission-line properties in a large sample of 744 type 1 AGNs. The sample includes Seyfert 1 galaxies and both radio-loud and radio-quiet quasars spanning an unprecedented wide range in both redshift $(0 \lesssim z \lesssim 5)$ and intrinsic luminosity ( $\sim 6$ orders of magnitude). The database and data processing will be described in detail in a future paper. A major concern regarding BEff studies is that selection effects in AGN samples, e.g. pertaining to emission-line strengths, might bias some measurements of the BEff. Our sample has the advantage of being drawn from a variety of surveys, with objects selected based on radio properties, grism spectroscopy, or broad-band color criteria. Hence, no specific selection criteria were applied for the data. Another key advantage is that our sample includes a wide range of luminosities at various redshifts. We can therefore address the separate redshift and luminosity dependences in the emission line data.

There are several interesting problems we wish to address. The most basic issue is to quantify the nature of empirical correlations with $L_{c}$ and/or $z$. In an upcoming study we will further examine correlations with other AGN properties such as radio-loudness (Baldwin 1977; Sargent, Steidel, \& Boksenberg 1989; Steidel \& Sargent 1991; Francis \& Koratkar 1995). We will also report on trends with $L_{c}$ or $z$ among various emission-line metallicity indicators. Here we present an initial analysis on the nature of the BEff. Our approach is to construct composite spectra for specific $L_{c}$ and $z$ intervals, thus providing high signal-to-noise spectra and allowing us to study trends in both weak and strong emission lines.

The AGN sample is described briefly in $\S 2$ and the calculation and analysis of the 
composite spectra in $\S 3$ and $\S 4$. The main results appear in several figures in $\S 5$. We compare our results with prior studies and discuss them in the context of suggested models in $\S 6$. Throughout this paper we use the cosmological parameters $\mathrm{H}_{o}=65 \mathrm{~km} \mathrm{~s}^{-1} \mathrm{Mpc}^{-1}$, $\Omega_{M}=0.3$, and $\Omega_{\Lambda}=0$ (Carroll, Press, \& Turner 1992). Introducing $\Omega_{\lambda}=0.7$ (Netterfield et al. 2002) instead of $\Omega_{\lambda}=0.0$, the luminosities at the highest redshifts would be $\sim 10 \%$ smaller, while at low redshifts they would reach a maximum of $\sim 20 \%$ larger at redshift $z \simeq 1$.

\section{The Quasar Sample}

We have compiled a large sample of rest-frame visible and ultraviolet spectra for 826 type 1 AGNs. A majority of the spectra were obtained by several groups for different studies over the last 20 years using ground-based instruments as well as International Ultraviolet Explorer (IUE) and Hubble Space Telescope (HST) (Bahcall et al. 1993; Baldwin et al. 1989; Chaffee et al. 1991; Corbin \& Boroson 1996; Kinney et al. 1991; Lanzetta, Turnshek, \& Sandoval 1993; Laor et al.1995; Sargent et al.1988,1989; Schneider, Schmidt, \& Gunn 1991a, b; Steidel 1990; Steidel \& Sargent 1991; Steidel unpublished; Storrie-Lombardi et al. 1996; Weymann et al. 1991, 1998; Wills et al. 1995; Zheng et al. 1997). In total, the sample contains 351 quasar spectra measured with $H S T$. Furthermore, we observed a large number of the quasars in our sample at redshifts $z \gtrsim 3$ (Constantin et al. 2002; Dietrich et al. 1999; Dietrich \& WilhelmErkens 2000; Dietrich et al.2002a,b). For this study we exclude Broad-Absorption Line quasars (BAL QSOs), although there are indications that their emission line properties do not differ from non-BAL quasars (Weymann et al.1991). We also excluded several quasar spectra with extremely poor signal-to-noise ratios. The sample we investigate consists of 744 type 1 AGNs.

Because this AGN sample was compiled from many independent projects, it encompasses a wide range of selection criteria. Most of the quasars especially at high redshifts were discovered by color selection techniques (e.g., Storrie-Lombardi et al.1996) or found by objective prism surveys (e.g., Chaffee et al.1991; Sargent et al.1988,1989; Schneider at al. 1991a, b).

We used the radio flux densities given in Véron-Cetty \& Véron (2001) to determine the radio loudness for the quasars. Following Kellermann et al. (1989) we calculated the parameter $R_{L}=\log \left(F_{R} / F_{B}\right)$ with $F_{R}$ as the radio flux density at $5 \mathrm{GHz}$ and $F_{B}$ as the Bband flux, each in the rest frame. Our classifications of radio-loud quasars are consistent with classifications available in the literature (Wills et al. 1995; Bischof \& Becker 1997; Wilkes

et al. 1999; Stern et al. 2000). In total our sample contains 265 radio-loud quasars and 479 
radio-quiet AGNs.

Kinney et al. (1990) and Pogge \& Peterson (1992) pointed out that a significant fraction of the scatter in the BEff in Seyfert 1 galaxies is caused by variability. They found that observations obtained at multiple epochs for individual sources display a BEff-like correlation (the intrinsic BEff), but with a significantly steeper slope than the BEff observed for an ensemble of AGNs. The physical mechanisms which drive each effect might be different, i.e., the ensemble BEff might be due primarily to variations in black hole mass while the intrinsic BEff might be caused by changes of the accretion rate $\dot{M}$ (Green 1999). For 8 of the 36 low luminosity AGNs $\left(\log \lambda L_{\lambda}(1450 \AA) \leq 44\right.$, for $\lambda L_{\lambda}$ in $\left.\operatorname{erg~s}^{-1}\right)$, the spectra are means from intense monitoring campaigns (Crenshaw et al. 1996; Collier et al. 1998,2001; Dietrich et al.1993; Goad et al.1999; Kaspi et al.1996; Korista et al.1995; Peterson et al.1994; Reichert et al. 1994; Santos-Lleó et al. 2001; Stirpe et al. 1994; Wanders et al. 1997). At the high luminosity end of the luminosity distribution, it has been shown that quasars vary on longer timescales and with smaller amplitudes (e.g., Kaspi et al. 2000). For these reasons, the scatter caused by intrinsic variability is not expected to strongly influence our analysis of the present large quasar sample, particularly when comparisons of composite spectra are employed.

We transformed each quasar spectrum to the rest-frame using a redshift measured from CIV $\lambda 1549$. To determine the redshift we fit a Gaussian profile to the upper part of the C IV $\lambda 1549$ emission line having $I_{\lambda} \geq 50 \%$ of the peak intensity. We corrected each AGN spectrum for Galactic extinction (Seaton 1979), based on the neutral hydrogen column den-

sity $N_{H}$ expressed in units of $10^{20} \mathrm{~cm}^{-2}$, assuming $E_{B-V}=N_{H} / 60$ (Dickey \& Lockman 1990).

The redshift distribution of the quasar sample is presented in Figure 1 as a function of continuum luminosity, $\lambda L_{\lambda}(1450 \AA)$. The quasars cover a redshift range of $0 \lesssim z \lesssim 5$ and nearly 6 orders of magnitude in luminosity. For most of the redshift range a reasonable coverage in luminosity is achieved, spanning at least 3 orders of magnitude for $z \gtrsim 1$. The radio-loud and radio-quiet quasars are displayed with different symbols in Figure 1. Their distributions across luminosity and redshift are very similar within out sample, although the fraction of radio-loud quasars increases for redshifts $z \lesssim 2$.

\section{Composite Spectra}

A common approach to study the BEff is to measure the emission line properties of individual quasars. However, the results are often limited especially for the weaker emission 
lines by the low signal-to-noise ratio of individual spectra. There can also be significant problems introduced by including upper limits on weak lines in individual spectra. To avoid those problems, we computed composite spectra in different intervals of redshift and luminosity. The analysis of these spectra has the advantage of more clearly representing the average properties of the sample used to calculate each composite. Figure 1 indicates the ranges in luminosity and redshift used to compute the composites. Except for the low and high luminosity ends of our sample, each composite spectrum is based on more than 20 individual quasar spectra. The scatter introduced by different individual spectra is taken into account by calculating a root-mean-square (standard deviation) spectrum for each composite, and using that to estimate the uncertainties in the measured equivalent widths (§4).

Before combining the individual quasar spectra, we normalized the flux densities to unity in a $20 \AA$ wide continuum range centered at $\lambda=1450 \AA$ by multiplying each spectrum with a suitable factor. We used these normalized quasar spectra to calculate the mean and rms spectrum for each composite, and the number of spectra contributing in each wavelength bin.

To avoid the influence of strong narrow absorption lines, we developed a search routine to detect strong narrow absorption features and exclude them from the calculation of the composite spectrum. To search for strong absorption lines we applied the following method (a more detailed description will be given in a subsequent paper on the properties of the entire quasar sample, Dietrich et al. 2002c). First, a preliminary mean spectrum is calculated for the sample of spectra under study. We then divided each individual spectrum by this preliminary mean to derive a ratio spectrum. Each ratio spectrum is smoothed with a running boxcar function, which provides in addition to the mean also an rms value for each wavelength box. The resulting rms spectrum indicates the scatter in flux for each wavelength element in the immediate spectral region. A narrow absorption line will cause a sharp increase in the rms spectrum. The contaminated spectral regions of individual spectra, identified by these rms spikes, are excluded from the calculation of the final composite spectrum, and no interpolation has been applied. Multiple tests with different AGNs and different sample sizes show that this technique sucessfully removes strong narrow absorption lines from our composite spectra (see below) without affecting the emission lines. Significant effects of narrow absorption lines remain only in the Ly $\alpha$ forest $(\lambda \lesssim 1216 \AA)$ for composites that include quasars with redshift $z \gtrsim 1$.

The resulting composite quasar spectrum for the entire sample is shown in Figure 2. A single power law fit to the continuum at $\lambda \geq 1200 \AA$ is also displayed; it has a slope of $\alpha=-0.43\left(F_{\nu} \propto \nu^{\alpha}\right)$. This spectral index is consistent with the slope reported for other composite spectra (Brotherton et al. 2001; Vanden Berk et al. 2001; Telfer et al. 2002). 
The bottom panel of Figure 2 shows the number of quasars contributing to each individual wavelength element. For every wavelength $800 \lesssim \lambda \lesssim 5600 \AA$ this composite quasar spectrum is based on at least $\sim 100$ quasars.

\section{Emission Line Measurements}

To measure the emission line fluxes we analyzed each composite spectrum with spectral fitting software provided by MIDAS ${ }^{1}$. We employed a powerlaw continuum, an ultraviolet and optical Fe-emission template, a Balmer continuum emission template, and multiple Gaussian profiles for the broad emission lines in multi-component fits using $\chi^{2}$ minimization. For the ultraviolet wavelength range we used the Fe emission template which Vestergaard \& Wilkes (2001) carefully derived from HST spectra of IZw1 (kindly provided by M. Vestergaard). The primary aim was to measure the broad and prominent Fe II-emission near Mg II $\lambda 2798$. However, we also used the UV template to correct for FeII and FeIII emission blended with C III] $\lambda 1909$ and other lines across the wavelength range $1400 \lesssim \lambda \lesssim 1900 \AA$. For the optical wavelength region around $\mathrm{H} \beta$ and $\mathrm{H} \gamma$, we used the empirical Fe II emission template obtained from IZw1 (kindly provided by T. Boroson; Boroson \& Green 1992). We adjusted the line widths in these Fe emission templates to match the measured widths of CIV $\lambda 1549$, $\operatorname{Mg} \operatorname{II} \lambda 2798$, or $\mathrm{H} \beta$.

We calculated several bound-free Balmer continuum emission spectra for $T_{e}=15000 \mathrm{~K}$, $n_{e}=10^{8}-10^{10} \mathrm{~cm}^{-3}$, and $0.1 \leq \tau_{\nu} \leq 2$ following the formalism suggested by Grandi (1982). These Balmer continuum emission templates were supplemented for $\lambda>3646 \AA$ with high order Balmer emission lines with $10 \leq n \leq 50$, i.e. H $\vartheta$ and higher (Storey \& Hummer 1995). The individual high-order Balmer lines were represented by Gaussian profiles and coadded.

The multi-component fit of a Balmer continuum, two Fe-emission templates, and a power law continuum yields independent measurements of the ultraviolet and optical Fe-emission strengths. Furthermore, the spectral index, $\alpha$, of the power law continuum does not include contributions from Balmer continuum or from broad Fe emission. The uncertainties associated with the fit of Balmer continuum emission and Fe emission templates were estimated from the $\chi^{2}$ distribution for each fit (cf., Dietrich et al. 2002a).

The emission line fluxes were measured after subtracting the power law continuum, Balmer continuum, and Fe emission spectra. The line fluxes were determined by multicomponent Gaussian fits. In general, we fit the emission lines with a "broad" and a "narrow"

\footnotetext{
${ }^{1}$ Munich Image Data Analysis System, trade-mark of the European Southern Observatory
} 
Gaussian component. For C IV $\lambda 1549$, however, it was necessary to introduce in addition a very broad component (which turned out to have typically FWHM $\simeq 10^{4} \mathrm{~km} \mathrm{~s}^{-1}$ ). To determine the fluxes of Ly $\alpha$ and $\mathrm{N} v \lambda 1240$, which are severely blended, we used the components obtained from the CIV $\lambda 1549$ profile as templates (preserving their widths and redshifts). Please see Figure 2 in Dietrich \& Hamann (2002d) for examples of fitted profiles. Note that most of the Ly $\alpha$ forest absorption on the blue side of the Ly $\alpha$ profile is corrected by the methode we applied to calculated the composite spectra. The OVI $\lambda 1034$ emission line is subject to severe corruption by $\operatorname{Ly} \alpha$ forest absorption. However, in composite spectra it can be assumed that the same fraction of the O vi $\lambda 1034$ emission line flux and the adjacent continuum flux is absorbed by the Ly $\alpha$ forest. Hence, the equivalent width is not expected to be significantly altered by Ly $\alpha$ forest absorption. The O vi $\lambda 1034$ emission line is also contaminated by Ly $\beta$ emission in the blue wing, whose relative strength shows a wide range, $\operatorname{Ly} \beta / \mathrm{O}$ vi $\lambda 1034=0.30 \pm 0.21$ (Laor et al. 1995). Our fits to the O vi $\lambda 1034$ emission line profiles were achieved with a broad and narrow Gaussian profile, and no additional component due to $\operatorname{Ly} \beta$ emission was necessary.

The C III] $\lambda 1909$ emission line complex was reconstructed with four gaussian profiles, corresponding to Al III $\lambda 1857$, Si III] $\lambda 1892$, and broad and narrow Gaussian profiles for C III] $\lambda 1909$. The correction for Fe emission allows a better measurement of Mg II $\lambda 2798$, He II $\lambda 1640$, N III] $\lambda 1750$, and the C III] emission line complex (Al III $\lambda 1857$, Si III] $\lambda 1892$, C III] $\lambda 1909)$. The measurement of the optical Fe II emission with an appropriate scaled template made it possible to measure the He II $\lambda 4686$ emission line which is otherwise severely blended with Fe II $(37,38)$ multiplets.

The cumulative errors in the emission line flux measurements and $W_{\lambda}$ were calculated as the square root of the sum of i) the uncertainties in the $\chi^{2}$ fitting and ii) the rms scatter that is introduced by both the noise and distribution of the individual spectra contributing to that particular composite spectrum, squared.

\section{Results and Analysis}

\subsection{Trends with Redshift}

To search for a dependence of emission line properties, particularly $W_{\lambda}$, on cosmic time (redshift), we selected a narrow luminosity range. The location of this luminosity range was also selected to achieve a comparable number of individual quasars contributing to each redshift bin in the $z-\lambda L_{\lambda}(1450 \AA)$ plane. To cover a redshift range as large as possible for an almost constant luminosity, we selected a luminosity range of $46.16 \leq \log \lambda L_{\lambda}(1450 \AA) \leq$ 
47.16 (i.e., $\left.43.0 \leq \log L_{\lambda}(1450 \AA) \leq 44.0\right)$. This range contains 323 quasars with $z \geq 0.5$ and an average luminosity of $\log \lambda L_{\lambda}(1450 \AA)=46.67 \pm 0.12$. We computed composite spectra for redshift bins with $\Delta z=0.5$ centered on redshift $z=0.75,1.25, \ldots, 3.75,4.25$, and 4.75 , respectively (Fig. 1). Figures $3 \mathrm{a}$ and $3 \mathrm{~b}$ show the composite spectra for each redshift bin. These composite spectra are normalized by the power law continuum which we derived from the multi-component fits in $\S 4$.

As a first impression the relative line strengths in the normalized composite quasar spectra stay nearly constant with redshift $z$. However, some emission lines show a marginal trend for increasing equivalent width at the highest redshifts. To quantify this result, we measured the equivalent width of most emission lines in the ultraviolet wavelength range $(\lambda \lambda 1000-3000 \AA)$ and of the broad ultraviolet Fe II-emission feature $(\lambda \lambda 2200-3090 \AA)$. The results for $W_{\lambda}$ as a function of redshift are displayed in Figures $4 \mathrm{a}$ and $4 \mathrm{~b}$. In general, the equivalent widths of the lines remain constant within $\sim 30 \%$. The stronger emission lines, however, like Ly $\alpha$, C IV $\lambda 1549$, and C III $] \lambda 1909$, show marginal trends for a relation of $W_{\lambda}$ and redshift. $W_{\lambda}$ stays nearly constant within less than $\sim 20 \%$ for redshifts less than $z \simeq 2-3$, but then for $z \gtrsim 3$ there are marginal indications for a slight increase of $W_{\lambda}$ at constant luminosity. Close inspection of Figures $4 \mathrm{a}$ and $4 \mathrm{~b}$ indicates that in particular the equivalent widths measured for the composites with $z \gtrsim 4$ are larger compared to lower redshifts. Most of the quasar spectra in our sample with $z \gtrsim 4$ were obtained by Constantin et al. (2002). They found evidence that in spite of strong contamination by Ly $\alpha$-forest absorption, Ly $\alpha$ is stronger for quasars with $z>4$ than in their low- $z$ counterparts.

\subsection{Trends with Luminosity}

Next we divided the quasar sample into eleven subsets with respect to the luminosity $L_{\lambda}(1450 \AA)$. Except for the lowest luminosity bin, each composite spectrum covers a range of $\Delta \log \lambda L_{\lambda}=0.5$ in luminosity starting at $\log \lambda L_{\lambda}(1450 \AA)=43.16 \mathrm{erg} \mathrm{s}^{-1}$ (Fig. 1). The two lowest luminosity composite spectra consist of less than 10 objects.

Figures $5 \mathrm{a}$ and $5 \mathrm{~b}$ show the composite spectra ordered by luminosity for the ultraviolet wavelength range $(\lambda \lesssim 2000 \AA)$, while Figure 6 shows the longer wavelengths $(\lambda \lambda 2000-$ $5600 \AA)$. Each composite spectrum is normalized by the power law continuum fit we derived from the multi-component analysis (§3). These normalized spectra show very obviously a strong relation between the equivalent width, $W_{\lambda}$, and the continuum luminosity represented by $\lambda L_{\lambda}(1450 \AA)$, i.e. the Baldwin Effect, for most of the emission lines. Figures $7 \mathrm{a}$ and $7 \mathrm{~b}$ show the measured values of $W_{\lambda}$ for each luminosity interval. The BEff can be seen easily in strong lines such as C IV $\lambda 1549$, O VI $\lambda 1034$, Ly $\alpha \lambda 1216$, C III] $\lambda 1909$, and Mg II $\lambda 2798$ (Figures 
$5 \mathrm{a}$ and 6). Taking advantage of the high signal-to-noise ratio of the composite spectra, we also detected this relation for weaker emission lines such as He II $\lambda 1640$. Furthermore, emission lines of the same ion but at different wavelengths (O III] $\lambda 1663$, [O III] $\lambda 5007$ and He II $\lambda 1640$, HeII $\lambda 4686$ ) possess nearly identical slopes (Tab. 1). For the first time, we report on the BEff for $\left.\mathrm{N}_{\mathrm{IV}}\right] \lambda 1486$, O III] $\lambda 1663$, and $\mathrm{N}$ III] $\lambda 1750$. In spite of the large scatter, the broad Fe emission features in the ultraviolet show some indications of a BEff, while within the errors, the optical Fe II emission lacks a BEff. In addition to the broad emission lines, we detect the Baldwin Effect also for the prominent forbidden emission line [O III] $\lambda 5007$ (Figure 6), which is regarded as a typical narrow-line region (NLR) emission line. In contrast to the strong BEff in most of the emission lines visible in Figures $5 \mathrm{a}$, 5b, and 6 , the Balmer lines $\mathrm{H} \gamma$ and $\mathrm{H} \beta$ show only weak indications of a Baldwin Effect, which might be restricted to the line center of the line profiles. Close inspection of the $\mathrm{Nv} \lambda 1240$ emission line (Figures 5a and 8) also indicates that this line does not exhibit a BEff like other high ionization lines. Lines having the same equivalent widths in each individual spectrum will cancel out in difference spectra (provided that their profiles are also the same), while lines whose equivalent widths are a function of luminosity will still be present in difference spectra. Note that N v $\lambda 1240$ has on average cancelled out in the difference spectra as shown in Figure 8. Hence, the difference spectra near $\mathrm{NV}$ and the careful deblending of the $\mathrm{Ly} \alpha-\mathrm{N} v \lambda 1240$ emission line complex both reveal that $\mathrm{N} v \lambda 1240$ remains nearly constant in $W_{\lambda}$ over $\sim 6$ orders of magnitude in continuum luminosity.

\subsection{Trends with Ionization Energy}

Zheng, Fang, \& Binette (1992), Espey et al. (1993), and Espey \& Andreadis (1999) have suggested that there is a correlation between the ionization energy, $\chi_{i o n}$, of a specific emission line and the strength (slope) of the BEff. In contrast to Espey \& Andreadis (1999), we use the ionization energy necessary to create the ion for collisionally excited lines like C IV $\lambda 1549$ and $\operatorname{Mg}$ II $\lambda 2798$, and the ionization energy needed to ionize the ion for recombination lines like Ly $\alpha$ and HeII.

The BEff slope of the lines under study shows a relatively close linear correlation with $\chi_{\text {ion }}$ (Figures 7a,b). We determined the slope $\beta$, where $\log W_{\lambda} \propto \beta \cdot \log \lambda L_{\lambda}(1450 \AA)$, of the BEff from a linear least square fit to $\log W_{\lambda}$ vs. $\log \lambda L_{\lambda}(1450 \AA)$. The fits were calculated taking into account the full uncertainties in $\log W_{\lambda}$. First, we calculated the slopes across the entire luminosity range, shown by the dotted line in Figures $7 \mathrm{a}$ and $7 \mathrm{~b}$. However, several of the emission lines indicate that the slope of the BEff is flatter at lower luminosities $\left(\log \lambda L_{\lambda}(1450 \AA) \lesssim 44\right)$. Therefore, we calculated additional linear fits for the luminosity 
range $\log \lambda L_{\lambda}(1450 \AA) \gtrsim 44$ (dashed-dotted lines). It is interesting to note that the lines showing a change in the BEff slope have high ionization energies with $\chi_{\text {ion }} \gtrsim 25-30 \mathrm{eV}$. The slopes of the $\log W_{\lambda}$ vs. $\log \lambda L_{\lambda}(1450 \AA)$ relations are listed in Table 1 and plotted against $\chi_{\text {ion }}$ in Figure 9 for the entire range (filled symbols) and for $\log \lambda L_{\lambda}(1450 \AA) \gtrsim 44$ (open symbols). Notice, that the slope of the BEff becomes steeper for increasing $\chi_{i o n}$. However, as has already been shown by earlier studies, N v $\lambda 1240$ deviates significantly from this trend (Espey \& Andreadis 1999). Nv $\lambda 1240$ stays constant or shows a slightly decreasing $\mathrm{W}_{\lambda}$ towards higher luminosity.

The slope $\beta$ for $\mathrm{O}$ vi $\lambda 1034$ is of particular interest for testing the hypothesis of a steeper BEff towards higher ionization energies since $\chi_{\text {ion }}\left(\mathrm{O}^{+5}\right)$ is more than twice $\chi_{\text {ion }}\left(H e^{+2}\right)$ and nearly 15 times $\chi_{\text {ion }}\left(\mathrm{Mg}^{+}\right)$. Assuming a constant ratio of $\mathrm{Ly} \beta / \mathrm{O}$ vi $\lambda 1034=0.30$ does not significantly change the slope of the BEff for Ovi $\lambda 1034$. However, $\beta(\mathrm{O}$ vi $\lambda 1034)$ would become steeper by nearly $25 \%$ if $\operatorname{Ly} \beta$ follows the same BEff as Ly $\alpha$ and $\operatorname{Ly} \beta / \operatorname{Ly} \alpha=0.059 \pm$ 0.04 (Laor et al. 1995).

To quantify the relationship of the BEff slopes $\beta$ to $\chi_{i o n}$, we calculated linear least square fits to the points in Figure 9 excluding the outlying data point for $\mathrm{N} v \lambda 1240$. The resulting slope, $\eta$, is the same with $(\eta=-0.00154 \pm 0.00024)$ or without $(\eta=-0.00150 \pm 0.00030)$ the O vi $\lambda 1034$ line included. This result indicates that O vi $\lambda 1034$ does not dominate the $\beta$ versus $\chi_{i o n}$ relation.

\section{Discussion}

\subsection{Comparisons with other Studies}

In general, the slopes of the Baldwin Effect derived here are consistent with prior investigations. However, the large range in both luminosity and redshift that distinguishes our quasar sample reveals correlations which were missed in some other studies. In particular, we can more clearly separate the trends with $L$ and $z$, and our use of composite spectra across a wide luminosity range more accurately probes the BEff slopes, e.g., for weaker lines. In the following we provide some more detailed comparisons with earlier work.

The slopes we derived for $\operatorname{Ly} \alpha \lambda 1216, \mathrm{C}$ IV $\lambda 1549, \mathrm{C} \operatorname{III}] \lambda 1909$, and $\operatorname{Mg} \operatorname{II} \lambda 2798$, are in good agreement with prior studies (Kinney et al. 1990; Osmer et al. 1994; Laor et al. 1995; Green 1996; Turnshek 1997; Zamorani et al. 1992; Zheng et al.1995; Wang et al. 1998; Espey \& Andreadis 1999). The steeper slopes for CIV $\lambda 1549$ in Osmer et al. (1994), Laor et al. (1995), and Green (1996), $\beta=-0.23$ compared to $\beta=-0.13$ here, might be caused by the luminosity range of their quasar samples, $\lambda L_{\lambda}(1450 \AA) \gtrsim 10^{44} \mathrm{erg} \mathrm{s}^{-1}$. Using only the 
$W_{\lambda}(\mathrm{C}$ IV $)$ measurements for $\lambda L_{\lambda}(1450 \AA) \gtrsim 10^{44} \mathrm{erg} \mathrm{s}^{-1}$ in our sample, we calculate a slope of the Baldwin Effect of $\beta=-0.20 \pm 0.03$ for $\mathrm{C} \operatorname{IV} \lambda 1549$ (Tab. 1).

A lack of the Baldwin Effect for C IV $\lambda 1549$ and C III] $\lambda 1909$ is reported by Wilkes et al. (1999). They conclude that this is caused by the Narrow-Line-Seyfert 1 galaxies (NLSy1s) in their sample which decrease the strength of the Baldwin Effect; a significant BEff is found for both CIV and CIII] if the NLSyls galaxies in their sample are omitted. We note that these NLSy1s are also low luminosity AGN. As can be seen in Figures 5a and 5b, the Baldwin relation is significantly flatter for low luminosities than at higher luminosities. Any AGN sample favoring a narrow luminosity range can dilute or bias the derived BEff. Similarly, the steeper BEff slopes reported by Laor et al. (1995), for O I $\lambda 1305$, C II $\lambda 1335$, Si IV $\lambda 1402$, and He II $\lambda 1640$ can be attributed to their smaller luminosity range $\left(\lambda L_{\lambda}(1350 \AA) \simeq 10^{45}-10^{48}\right.$ $\left.\operatorname{erg} \mathrm{s}^{-1}\right)$.

The slope for the Baldwin Effect for the high ionization emission line O VI $\lambda 1034\left(\chi_{\text {ion }} \simeq\right.$ $114 \mathrm{eV})$ is also consistent with prior investigations. The reported slope $\beta(\mathrm{O}$ vi $\lambda 1034)$ varies between $-0.30 \pm 0.04$ (Zheng et al. 1995; Turnshek 1997) and $-0.15 \pm 0.08$ (Laor et al. 1995), with most recent studies favoring a less steep anti-correlation $(-0.18 \pm 0.03$; Espey \& Andreadis 1999). Wilkes et al. (1999) found only a marginal anti-correlation of $W_{\lambda}(\mathrm{O}$ vi $\lambda 1034)$ and continuum luminosity. However, they noted that this result is based on just ten objects. Green (1996) and Green et al. (2001) did not detect a significant Baldwin Effect for O VI $\lambda 1034$ in their analysis of the Large Bright QSO Survey (LBQS) data set, taking all quasar spectra into account, i.e. including upper limit measurements as well. However, using only those spectra with detected O vi $\lambda 1034$ the Baldwin Effect is clearly present. A possible reason for the missing Baldwin Effect for O vi $\lambda 1034$ in their study might be the very narrow luminosity range $\left(46.2 \lesssim \log \lambda L_{\lambda}(2500 \AA) \lesssim 47.0\right)$ covered by LBQS quasars at these wavelengths. A comparison of our Figure 7a with Figure 2 in Green et al. (2001) shows that we measure the same range of O vi $\lambda 1034$ equivalent widths for this luminosity, $\log W_{\lambda}(\mathrm{O}$ vi $\lambda 1034) \simeq 1.1$. Hence, we conclude that the non-detection of the Baldwin Effect for this high-ionization line in Green et al. (2001) is predominatly caused by their very small luminosity range.

In contrast to Wills et al. (1999), we detect a significant Baldwin Effect for the prominent NLR emission line [O III] $\lambda 5007$ (Fig. 7b). The slope $\beta$ of the anti-correlation is nearly identical to the one which we derived for O III] $\lambda 1663$ (Tab. 1, Fig. 9). Again, the lack of a BEff for [O III $]$ A5007 in Wills et al. (1999) might be caused by the covered luminosity range of less than two orders of magnitude.

In agreement with prior investigations, we found no significant Baldwin Effect for N vi1240 (Osmer et al. 1994; Laor et al.1995; Turnshek 1997; Espey \& Andreadis 1999). 
Instead of a decreasing equivalent width for increasing continuum strength, $W_{\lambda}\left(\mathrm{N}_{\mathrm{v}} 1240\right)$ stays nearly constant, even though it has a high value of $\chi_{\text {ion }}=78 \mathrm{eV}$.

\subsection{Comparison with Model Predictions}

Although much observational and theoretical effort has been spent to decipher the process which causes the Baldwin Effect, the mechanism is still unclear. Several models have been suggested which emphasize different aspects, i.e., contributions from optically thin clouds and luminosity-dependent ionization parameter, covering factor, ionizing continuum shape, and chemical composition of the gas.

\subsubsection{Luminosity Dependent Ionization and Covering Factor}

The dependence of the strength of the Baldwin Effect on the ionization energy indicates that a decreasing covering factor towards higher luminosities is not sufficient to explain the observed BEff for each emission line. To explain the observed BEff for CIV $\lambda 1549$, Mushotzky \& Ferland (1984) presented model calculations suggesting that the BEff is caused by a decrease of the ionization parameter, $U$, as well as the covering factor towards higher luminosities. Although this model predicts a weaker Baldwin Effect for lower luminosity AGN, it also predicts that Ly $\alpha$ will lack a BEff or show only a weak BEff. A luminosity dependence of the covering factor might be indicated independently by measurements of X-ray absorption (e.g., Lawrence \& Elvis 1982).

A variant of this picture was outlined by Shields, Ferland, \& Peterson (1995), who suggested that the luminosity dependence could be caused by a luminosity-dependent covering factor of optically thin clouds which would emit preferentially high-ionization lines. The presence of this component in the BLR is suggested from several aspects of variability in Seyfert galaxies, as well as other arguments. While luminosity dependence of coverage by high-ionization clouds in the BLR is apparently still a viable scenario for understanding the BEff, this interpretation suffers from the lack of a strong physical basis for predicting this behavior; explanations are ad hoc or phenomenological at best.

\subsubsection{Luminosity dependent Spectral Energy Distribution}

Several models have been advanced that focused primarily on the spectral energy distribution (SED) of the ionizing continuum, and its consequences. This approach was motivated 
by increasing evidence that the SED of the continuum becomes softer for more luminous AGN (e.g., Sargent \& Malkan 1982; Binette et al. 1989; Schulz 1992; Netzer et al. 1992; Zheng et al. 1992; Zheng \& Malkan 1993; Green 1996, 1998; Wang et al. 1998).

Empirical models have been suggested which combine a powerlaw continuum and a thermal UV bump. Detailed investigations of X-ray and ultraviolet continuum observations, i.e., the overall ionizing continuum shape, yield indications that the SED becomes softer for increasing luminosity. In particular, it has been shown that $\alpha_{o x}$, the two-point power index which connects the ultraviolet at $2500 \AA$ to the X-ray continuum at $2 \mathrm{keV}$, increases significantly with luminosity (Tananbaum et al. 1986; Wilkes et al. 1994; Green et al. 1995). This softening can be understood by a shift of the thermal UV-bump towards longer wavelengths in more luminous quasars, i.e., $L_{u v}$ increases more than $L_{x}$ for increasing luminosity resulting in a greater $\alpha_{o x}$ (cf., Binette et al. 1989; Zheng \& Malkan 1993).

Early models of geometrically thin and optically thick accretion discs focused on the consequences of the disc inclination and the location of gas relative to the disc (Netzer 1985, 1987). Although these models can not explain the BEff for several orders of magnitude in luminosity they provide an explanation for scatter in the $L_{c}$ vs. $W_{\lambda}$ relation at a given luminosity. The spectral energy distribution (SED) of the accretion disc continuum depends on the luminosity which is in turn related to the mass of the black hole and the accretion rate. These dependencies were suggested as the primary mechanism to cause the Baldwin Effect. Recently, Wandel (1999a,b) presented an evolutionary scenario which connects the growth of the black hole mass, the accretion rate and the continuum luminosity. As the mass of the black hole increases and consequently also the luminosity, the peak temperature of the UV bump decreases. This results in a shift of the peak of the thermal UV bump to longer wavelengths, i.e., the continuum becomes softer. Netzer et al. (1992) calculated the $\log W_{\lambda}(\operatorname{Ly} \alpha)$ vs. $\log L_{\lambda}(1216 \AA)$ relation for a wide range of black hole masses, accretion rates, and inclination angles in geometrically thin accretion disc models. The spread of the calculated $W_{\lambda}(\operatorname{Ly} \alpha)$ at a given luminosity is mainly caused by the different inclination angles of the accretion disc. Assuming that our composite spectra represent an average over a variety of disc inclinations, the BEff which we measured for Ly $\alpha$ is consistent with their model predictions.

The observed ionization dependence of the Baldwin Effect provides strong evidence for a luminosity dependent SED of the ionizing continuum and thus favors accretion disc models. However, the detailed models discussed by Wandel (1999b) show that the SEDs of accretion discs depend in a complex way on the mass of the black hole and the accretion scenario. In particular, the cutoff energy of the thermal UV-bump is expected to depend on the black hole mass. These models also predict that luminous quasars with massive black 
holes should show low cutoff energies, while Seyfert 1 galaxies with intermediate massive black holes should display high cutoff energies. The low luminosity NLSy1 galaxies with low mass black holes should also exhibit high cutoff energies. Different accretion scenarios would introduce additional scatter to this relation.

In summary, accretion disc models provide a reasonable explanation of the Baldwin Effect, in particular for the ionization energy $\chi_{i o n}$ dependent strength. Although these models indicate that the luminosity-dependent SED is a major mechanism which drives the BEff, the large possible parameter space allows for additional effects. Evidence for effects in addition to variations of the spectral energy distribution is given by the failure to explain the lack of a Baldwin Effect for another high ionization emission line, Nv $\lambda 1240$.

\subsubsection{The Influence of Metallicity Variations}

Baldwin et al. (1995) and Korista et al. $(1997,1998)$ introduced the "locally optimallyemitting cloud" (LOC) model, which assumes that the BLR cloud ensemble covers a wide range of internal densities and occurs over a wide range in distance from the central continuum source. Under these conditions the emitted spectrum is controlled by powerful selection effects and the typical observed quasar spectrum can be naturally produced (Korista et al. 1997, 1998).

Korista et al. (1998) computed for a wide range of different continuum slopes $\left(F_{\nu} \propto\right.$ $\left.\nu^{\alpha},-2<\alpha<-1\right)$, densities $\left(n_{e}=10^{8}\right.$ to $10^{12} \mathrm{~cm}^{-3}$ ), and metallicities (0.2 to 10 times solar) the resulting emission line spectra. In recent years there has been growing evidence that the chemical composition of quasar gas can reach several times solar metallicity with a trend of higher metallicities in more luminous quasars (Hamann \& Ferland 1993, 1999; Ferland et al.1996, Dietrich et al.1999; Dietrich \& Wilhelm-Erkens 2000). The emission lines studied by Korista et al. (1998) follow the general trend for $W_{\lambda}$ diminishing with softer continuum shapes. Assuming the softer continua are related to higher luminosities, this yields the Baldwin Effect. However, in contrast to those emission lines, N v $\lambda 1240$ lacks a Baldwin Effect even though its high ionization energy $\chi_{i o n}=78 \mathrm{eV}$ suggests that the decrease of $W_{\lambda}(\mathrm{Nv})$ should be strong. Korista et al. (1998) introduced metallicity as an additional parameter to provide a possible explanation for the lack of a Baldwin Effect in N v $\lambda 1240$. They found in their calculations that the equivalent width of Ly $\alpha, \operatorname{CIV} \lambda 1549$, or O vi $\lambda 1034$ show only a weak dependence on the gas metallicity, but N v $\lambda 1240$ strongly depends on it.

In particular, they assume that nitrogen scales like a secondary element, so that its abundance relative to the other metals increases linearly with the overall metallicity (e.g., N/O 
$\propto \mathrm{O} / \mathrm{H}$, see Hamann et al. 2002). Korista et al. (1998) assume that the metallicity increases with increasing AGN luminosity (Hamann \& Ferland 1999). With this abundance behavior,

Korista et al. (1998) showed that the equivalent width of $\mathrm{N} v \lambda 1240$ is not expected to display a BEff. Therefore, the decreasing equivalent width that should occur in Nv $\lambda 1240$ as part of the normal BEff is approximately compensated by the increasing relative abundance of nitrogen. The net result is that Nv $\lambda 1240$ shows essentially no BEff.

Overall, the BEff slopes that we derived for the emission lines in our quasar sample are in good agreement with the predictions by Korista et al. (Table 1). The observed slopes of C IV $\lambda 1549$ and He II $\lambda 1640$ are smaller than expected, while the slope in N III] $\lambda 1750$ is steeper. However, if only measurements with $\lambda L_{\lambda}(1450 \AA) \gtrsim 10^{44} \mathrm{erg} \mathrm{s}^{-1}$ are taken into account, the observations yield a comparable steep anti-correlation as predicted by Korista et al. (1998). We will discuss these comparisons further in a forthcoming paper on the trends in metallicity in this dataset (see also $\S 6.4$ below).

\subsection{Ionization Dependence and Curvature of the Baldwin Effect}

A common prediction of models that involve a softer continuum SED towards higher luminosities is a steeper slope of the Baldwin Effect for lines with increasing ionization energy $\chi_{i o n}$ (Zheng et al. 1992; Zheng \& Malkan 1993; Korista et al. 1998; Wandel 1999a). Figures $7 \mathrm{a}, \mathrm{b}$ and 9 provide clear evidence for this prediction. High ionization lines like C IV $\lambda 1549$, HeII $\lambda 1640$, and He II $\lambda 4686$, and especially O VI $\lambda 1034$ are particularly valuable. These emission lines respond to photoionizing energies of $\sim 48 \mathrm{eV}, \sim 54 \mathrm{eV}$, and $\sim 114 \mathrm{eV}$, respectively. Hence, they can be used to probe the location of the cutoff energy of the thermal UV-bump. The strong BEff in these high-ionization lines is expected if the ionizing continuum becomes softer with increasing continuum luminosity. In this case the relative number of ionizing photons with $h \nu \gtrsim 50 \mathrm{eV}$ decreases as the UV bump is moving towards longer wavelengths. While there is only a marginal trend for a slightly steeper Baldwin Effect for HeII than for CIV, the higher ionization O VI $\lambda 1034$ line displays a significantly stronger BEff (Fig. 9). The decrease of the relative number of ionizing photons with a softer continuum, caused by the shift of the UV bump to longer wavelengths, results in weaker high-ionization lines compared to lower ionization ones. The shift of the UV bump to longer wavelengths will affect emission lines at higher ionization energy first. In addition, the relative continuum strength beneath the emission lines will be enhanced, starting at short ultraviolet wavelengths. Both effects together result in smaller $W_{\lambda}$ for higher luminosities.

In addition to the general trend of steeper BEff for higher $\chi_{i o n}$, the curvature or flattening of the BEff towards lower luminosities can be accommodated in the framework of some of the 
luminosity-dependent SED models (e.g., Netzer et al. 1992; Wandel 1999a). The flattening at low luminosities has led to suggestions that Seyfert 1 nuclei do not participate in the Baldwin Effect but have instead equivalent widths independent of luminosity (e.g., Wampler et al. 1984). A close inspection of Figures 7a,b provides some evidence for the tendency of a flatter slope $\beta$ of the BEff at low luminosities in comparison to higher luminosities, as can be seen for several emission lines (Si IV $\lambda 1402$, N IV] $\lambda 1486$, C IV $\lambda 1549$, He II $\lambda 1640$, O III] $\lambda 1663$, $\mathrm{N}$ III] $\lambda 1750$, He II $\lambda 4686)$. The turnover appears to occur near $\lambda \log L_{\lambda}(1450 \AA) \simeq 44$, in quite good agreement with predictions (Wandel 1999a). Within this context it is interesting to note that the steepening of the Baldwin Effect for $\log \lambda L_{\lambda}(1450 \AA) \simeq 44$, is observed for emission lines with $\chi_{\text {ion }} \gtrsim 25-30 \mathrm{eV}$.

\subsection{Individual emission line pairs}

For several elements we have measured more than one emission line strength. The comparison of the strength of the Baldwin Effect may provide information about the emission line region and the mechanism which causes the observed anti-correlation of $W_{\lambda}$ and continuum luminosity.

For hydrogen we have measured two Balmer emission lines $(\mathrm{H} \gamma, \mathrm{H} \beta)$ and Ly $\alpha$. The slope of the Baldwin Effect of $\mathrm{H} \gamma$ and $\mathrm{H} \beta$ is very similar and within the uncertainties consistent with zero, i.e. no Baldwin Effect for Balmer lines. The missing Baldwin Effect for these lines provide some argument against a luminosity dependent covering factor, at least for the gas component which is the dominant contributor to these emission lines. While the equivalent width $W_{\lambda}(\mathrm{H} \beta)$ remains nearly constant, the flux ratio $\mathrm{Ly} \alpha / \mathrm{H} \beta$ decreases by a factor $\sim 1.5$ from low to high luminosities $(\sim 8.5$ to $\sim 5.5)$. The different behaviour of the Balmer lines and Ly $\alpha$ might be also related to the complex physical processes of Ly $\alpha$ and $\mathrm{H} \beta$ emission (Netzer et al. 1995). The prominent BEff of Ly $\alpha$ and the lack of a BEff for $\mathrm{H} \beta$ may be caused in part by a relative stronger increase of the local continuum beneath Ly $\alpha$ compared to the optical wavelength range of $\mathrm{H} \beta$. However, Ly $\alpha$ shows a significant BEff which fits well in the slope $\beta$ vs. $\chi_{\text {ion }}$ relation (Fig. 7a, 9). Ly $\alpha$ might be blended with He II $\lambda 1216$ and O v] $\lambda 1218$ (Ferland et al. 1992; Shields et al. 1995). For standard BLR conditions the contribution of He II $\lambda 1216$ and $\mathrm{OV}] \lambda 1218$ are negligible (less than $\sim 10 \%$, priv.comm. K. Korista). But for higher densities and higher ionization parameter $\mathrm{U}$, particularly for optically thin conditions, these emission lines can be quite strong compared to Ly $\alpha$ (Shields et al. 1995). Because $\mathrm{O} \mathrm{v}] \lambda 1218$ has an ionization energy of $\chi_{i o n}=77.4 \mathrm{eV}$, this emission line might show a strong BEff. If optically thin gas is a significant fraction of the BLR gas, it might be possible that the moderate BEff of Ly $\alpha$ might be caused at least in part by $\mathrm{O} v] \lambda 1218$. However, 
if $\mathrm{O} \mathrm{v}] \lambda 1218$ is a significant contamination of $\mathrm{Ly} \alpha$ it is expected from the results in Shields et al. (1995) that He II $\lambda 1640$ and C IV $\lambda 1549$ should be stronger than observed. In addition, variability behavior in some objects suggests that the optically thin gas may contribute most strongly to the line wings (e.g., Ferland et al. 1990; Peterson et al. 1993), but the part of the emission lines that changes the most with luminosity has a narrow FWHM (e.g., Osmer et al. 1994). Although we cannot exclude that O v $] \lambda 1218$ provides some contribution to Ly $\alpha$, it does appear that Ly $\alpha$ itself shows a moderate Baldwin Effect in contrast to the Balmer emission lines.

The two emission lines of $\mathrm{He}^{+}$which we measured, HeII $\lambda 1640$ and HeII $\lambda 4686$, show very similar slopes for the Baldwin Effect. This behavior is expected for He II $\lambda 1640$ and He II $\lambda 4686$ as typical recombination lines. The similar slope of the BEff provides also some evidence that luminosity-dependent radiative transfer effects, collisional effects or dust effects can not affect these emission lines.

Another line pair we measured is $\mathrm{O}$ III $] \lambda 1663$ and $[\mathrm{O}$ III $] \lambda 5007$. While the ionization energy necessary to create $\mathrm{O}^{+2}$ is $\chi_{i o n}=35.1 \mathrm{eV}$, the excitation energies of these lines differ by a factor of $\sim 3$. If the ionizing continuum becomes softer for increasing luminosity, the temperature in the emission line region should drop and hence O III] $\lambda 1663$ should be more affected than [O III $] \lambda 5007$. Therefore, we might expect O III $] \lambda 1663$ to have a steeper BEff than $[\mathrm{O}$ III $] \lambda 5007$. Observationally their BEffs are the same. However, the comparison of these two lines is very complicated because they differ in critical density by several orders of magnitudes $\left(n_{e}^{\text {crit }} \simeq 7 \times 10^{5} \mathrm{~cm}^{-3}\right.$ for $[\mathrm{O} \mathrm{III}] \lambda 5007$ and $n_{e}^{\text {crit }} \simeq 3 \times 10^{10} \mathrm{~cm}^{-3}$ for $\mathrm{O}$ III $\left.] \lambda 1663\right)$. In particular, $[\mathrm{O}$ III] $] 55007$ forms in the narrow line region (NLR), which might be heated and ionized additionally by shocks, and is, in any case, spatially distinct from the BLR. The different kinematics of the O III emitting gas is also given by the significantly different profiles we used to measure the emission line flux and equivalent width. The OIII] $\lambda 1663$ emission line was fit with a broad and narrow Gaussian profile (with an average FWHM of $5140 \pm 460 \mathrm{~km} \mathrm{~s}^{-1}$ and $1865 \pm 290 \mathrm{~km} \mathrm{~s}^{-1}$, respectively). The two Gaussian components we used to fit [O III] $\lambda 5007$ result in a profile different compared to O III] $\lambda 1663$, with average FWHMs of $1490 \pm 50 \mathrm{~km} \mathrm{~s}^{-1}$ and $480 \pm 25 \mathrm{~km} \mathrm{~s}^{-1}$. The overall average FWHM of the fitted O III] $\lambda 1663$ and [O III] $\lambda 5007$ lines are $4130 \pm 450 \mathrm{~km} \mathrm{~s}^{-1}$ and $725 \pm 100 \mathrm{~km} \mathrm{~s}^{-1}$, respectively.

We have studied three emission lines of nitrogen. N v $\lambda 1240$ lacks within the errors a BEff. In contrast, the intercombination lines $\mathrm{N}$ IV $] \lambda 1486$ and $\mathrm{N}$ III $] \lambda 1750$ exhibit a Baldwin Effect that is consistent with the overall trend of $\beta$ vs. $\chi_{\text {ion }}$ (Fig. 9). It might be possible that additional effects influence the emission properties of these nitrogen intercombination lines at higher luminosities. We will discuss these issues in our forthcoming paper on elemental abundances. 


\section{Conclusion}

We have investigated a large sample of 744 type 1 AGN covering the redshift range from $0 \leq z \leq 5$ and nearly 6 orders of magnitude in continuum luminosity. To enhance the signalto-noise ratio, minimize the influence of peculiarities of individual quasars, and investigate weak as well as strong emission lines, we computed composite spectra representing narrow intervals in redshift and luminosity. The emission line fluxes were derived using multicomponent Gaussian fits after removing a powerlaw continuum fit, a Balmer continuum emission template, and a UV and optical iron emission template from the composite spectra. Our main results are the following.

- In composite spectra spanning the full redshift range at nearly constant luminosity we detect no strong trend in the line $W_{\lambda}$ with redshift i.e., with cosmic time. However, there is a marginal tendency for the highest redshift quasars $(z \gtrsim 4)$ to show slightly stronger emission lines than their counterparts at lower redshift.

- In the composite spectra ranked by luminosity we find a significant Baldwin Effect in nearly all emission lines in the ultraviolet to optical domain. The only exceptions are $\mathrm{Nv} \lambda 1240, \mathrm{H} \beta, \mathrm{H} \gamma$, and optical Fe II which remain constant in $W_{\lambda}$ within the uncertanties. The lack of a BEff for the high-ionization feature NV $\lambda 1240$ suggests that the chemical composition of the gas is an additional parameter that can strongly influence the equivalent width of this and possibly other lines.

- We detect a strong Baldwin Effect for the prominent NLR emission line [O III] $]$ 5007. The strength of the BEff for [O III $] \lambda 5007$ is very similar to the BEff measured in O III] $\lambda 1663$.

- The slope $\beta$ of the Baldwin Effect, where $\log W_{\lambda} \propto \beta \cdot \log \lambda L_{\lambda}$, shows a significant correlation with the ionization energy, $\chi_{i o n}$, needed to produce the lines.

- The slope of the Baldwin Effect, $\beta$, tends to be steeper at higher luminosities, $\lambda L_{\lambda}(1450 \AA) \gtrsim$ $10^{44} \mathrm{erg} \mathrm{s}^{-1}$, compared to the lower luminosity regime.

- The Baldwin Effect, its steepening towards higher luminosities, and the correlation of the slope $\beta$ with $\chi_{\text {ion }}$ can all be well explained in the context of a luminosity dependent spectral energy distribution of the ionizing continuum. Assuming that the SED can be described as a combination of a powerlaw continuum and a thermal UV bump, the ionizing continuum becomes softer for increasing luminosity as the UV bump is shifted to longer wavelengths. This behaviour can be explained with accretion disc models as suggested by Netzer et al. (1992) and Wandel (1999a,b). 
We are grateful to numerous colleagues for providing many of these spectra in digital form, in particular J.A. Baldwin, T.A. Boroson, M.R. Corbin, A. Laor, W.L.W. Sargent, D.P. Schneider, C.C. Steidel, R.J. Weymann, and W. Zheng. We also thank Desika Narayanan for reducing the spectra obtained at Lick Observatory. MD and FH have benefited from support from NASA grant NAG 5-3234 and NSF through AST-99-84040. MV greatfully acknowledges financial support from the Columbus Fellowship.

\section{REFERENCES}

Bahcall, J.N., Bergeron, J., Boksenberg, A., et al. 1993, ApJS, 87, 1

Baldwin, J.A. 1977, ApJ, 214, 679

Baldwin, J.A., Wampler, E.J., \& Gaskell, C.M. 1989, ApJ, 338, 630

Baldwin, J.A., Ferland, G.J., Korista, K., \& Verner, D. 1995, ApJ, 455, L119

Baldwin, J.A. 1999, in "Quasars and Cosmology", ASP Conf.Ser. 162, eds. G. Ferland \& J.A. Baldwin, p. 475

Binette, L., Prieto, A., Szuszkiewicz, E., \& Zheng, W. 1989, ApJ, 343, 135

Bischof, O.B. \& Becker, R.H. 1997, AJ, 113, 2000

Boroson, T.A. \& Green, R.F. 1992, ApJS, 80, 109

Brotherton, M.S., et al. 2001, ApJ, 546, 775

Carroll, S.M., Press, W.H., \& Turner, E.L. 1992, ARA\&A, 30, 499

Cen, R. \& Ostriker, J.P. 1999, ApJ, 519, L109

Chaffee, F.H., Foltz, C.B., Hewett, P.C., et al. 1991, AJ, 102, 461

Collier, S., et al. 1998, ApJ, 500, 162

Collier, S., et al. 2001, ApJ, 561, 146

Constantin, A., Shields, J.C., Hamann, F., Foltz, C.B., \& Chaffee, F.H. 2002, ApJ, 565, 50

Corbin, M.R. \& Boroson, T.A. 1996, ApJS, 107, 69

Crenshaw, D.M., et al. 1996, ApJ, 470, 322 
Dickey, J.M. \& Lockman, F.J. 1990, ARA\&A, 28, 215

Dietrich, M., et al. 1993, ApJ, 408, 416

Dietrich, M., et al. 1999, A\&A, 352, L1

Dietrich, M. \& Wilhelm-Erkens, U. 2000, A\&A, 354, 17

Dietrich, M., Appenzeller, I., Vestergaard, M., \& Wagner, S. 2002a, ApJ, 564, 581

Dietrich M., Hamann F., Appenzeller I., Vestergaard M., \& Wagner S.J. 2002b, in ESO Astrophysics Symposia "Lighthouses of the Universe: The Most Luminous Celestial Objects and their use for Cosmology", eds. R. Sunyaev, et al., in press

Dietrich, M. \& Hamann, F. 2002c, in prep.

Dietrich, M. \& Hamann, F. 2002d, in prep.

Espey, B.R., Lanzetta, K.M., Turnshek, D.A. 1993, BAAS, 25, 1448

Espey, B.R. \& Andreadis, S.J. 1999, in "Quasars and Cosmology", ASP Conf.Ser. 162, eds. G. Ferland \& J.A. Baldwin, p.351

Ferland, G.J., Korista, K.T., \& Peterson, B.M. 1990, ApJ, 363, L21

Ferland, G.J., Peterson, B.M., Horne, K., Welsh, W.F., \& Nahar, S.N. 1992, ApJ, 387, 95

Ferland, G.J., Baldwin, J.A., Korista, K.T., et al. 1996, ApJ, 461, 683

Forster, K., Green, P.J., Aldroft, T., Vestergaard, M., Foltz, C.B., \& Hewett, P.C. 2001, ApJS, 134, 35

Francis, P.J. \& Koratkar, A.P. 1995, MNRAS, 274, 504

Goad, M., et al. 1999, ApJ, 524, 707

Granato, G.L., Silva, L., Monaco, P., et al. 2001, MNRAS, 324, 757

Grandi, S.A. 1982, ApJ, 255, 25

Green, P.J., Schartel, N., Anderson, S.F., et al. 1995, ApJ, 450, 51

Green, P.J. 1996, ApJ, 467, 61

Green, P.J. 1998, ApJ, 498, 170 
Green, P.J. 1999, in "Quasars and Cosmology", ASP Conf.Ser. 162, eds. G. Ferland \& J.A. Baldwin, p.265

Green, P.J., Forster, K., \& Kuraszkiewicz, J. 2001, ApJ, 556, 727

Hamann, F. \& Ferland, G.J. 1993, ApJ, 418, 11

Hamann, F. \& Ferland, G.J. 1999, ARA\&A, 37, 487

Hamann, F., Korista, K.T., Ferland, G.J., Warner, C., \& Baldwin, J.A. 2002, ApJ, 564, 592

Kaspi, S., et al. 1996, ApJ, 470, 336

Kaspi, S., Smith, P.S., Netzer, H., et al. 2000, ApJ, 533, 631

Kauffmann, G. \& Haehnelt, M.G. 2000, MNRAS, 311, 576

Kellermann, K.I., Sramek, R., Schmidt, M., Shaffer, D.B., \& Green, R. 1989, AJ, 98, 1195

Kinney, A.L., Rivolo, A.R., \& Koratkar, A.P. 1990, ApJ, 357, 338

Kinney, A.L., Bohlin, R.C., Blades, J.C., \& York, D.G. 1991, ApJS, 75, 645

Korista, K.T., et al. 1995, ApJS, 97, 285

Korista, K.T., Baldwin, J.A., \& Ferland, G.J. 1997, ApJS, 108, 401

Korista, K.T., Baldwin, J.A., \& Ferland, G.J. 1998, ApJ, 507, 24

Lanzetta, K.M., Turnshek, D.,A., \& Sandoval, J. 1993, ApJS, 84, 109

Laor, A., Bahcall, J.N., Jannuzi, B.T., Schneider, D.P., \& Green, R.F. 1995, ApJS, 99, 1

Lawrence, A. \& Elvis, M. 1982, ApJ, 256, 410

Mushotzky, R. \& Ferland, G.J. 1984, ApJ, 278, 558

Netterfield, C.B., et al. 2002, ApJ, 571, 604

Netzer, H. 1985, MNRAS, 216, 63

Netzer, H. 1987, MNRAS, 225, 55

Netzer, H., et al. 1995, ApJ, 448, 27

Netzer, H., Laor, A., \& Gondhalekar, P.M. 1992, MNRAS, 254, 15 
Osmer, P.S., Porter, A.C., \& Green, R.F. 1994, ApJ, 436, 678

Osmer, P.S. \& Shields, J.C. 1999, in "Quasars and Cosmology", ASP Conf.Ser. 162, eds. G. Ferland \& J.A. Baldwin, p.235

Peterson, B.M., et al. 1993, ApJ, 402, 469

Peterson, B.M., et al. 1994, ApJ, 425, 622

Pettini, M. 1999, in Proc.of ESO Workshop "Chemical Evolution from Zero to High Redshift", ed. J. Walsh \& M. Rosa, LNP, p.233

Pogge, R.W. \& Peterson, B.M. 1992, AJ, 103, 1084

Reichert, G.A., et al. 1994, ApJ, 425, 582

Santos-Lleó, M., et al. 2001, A\&A, 369, 57

Sargent, W.L.W. \& Malkan, M.A. 1982, ApJ, 254, 22

Sargent, W.L.W., Boksenberg, A., \& Steidel, C.C. 1988, ApJS, 68, 539

Sargent, W.L.W., Steidel, C.C., \& Boksenberg, A. 1989, ApJS, 69, 703

Schneider, D.P., Schmidt, M., \& Gunn, J.E. 1991a, AJ, 101, 2004

Schneider, D.P., Schmidt, M., \& Gunn, J.E. 1991b, AJ, 102, 837

Schulz, H. 1992, in "Physics of Active Galactic Nuclei", eds. W.J. Duschl \& S.J. Wagner (Berlin: Springer), p.235

Seaton, M.J. 1979, MNRAS, 187, 73

Shields, J.C., Ferland, G.J., \& Peterson, B.M. 1995, ApJ, 441507

Steidel, C.C. 1990, ApJS, 74, 37

Steidel, C.C. \& Sargent, W.L.W. 1991, ApJ, 382, 433

Stern, D., Djorgovski, S.G., Perley, R.A., de Carvalho, R.R., \& Wall, J.V. 2000, AJ, 119, 1526

Stirpe, G.M., et al. 1994, ApJ, 425, 609

Storey, P.J. \& Hummer, D.G. 1995, MNRAS, 272, 41 
Storrie-Lombardi, L.J., McMahon, R.G., Irwin, M.J., \& Hazard, C. 1996, ApJ, 468, 121

Tananbaum, H., Avni, Y., Green, R.F., Schmidt, M., \& Zamorani, G. 1986, ApJ, 305, 57

Telfer, R.C., Zheng, W., Kriss, G.A., \& Davidsen, A.F. 2002, ApJ, 565, 773

Turnshek, D.A. 1997, in ASP Conf.Ser.128, "Mass Ejection from AGN", eds. N. Arav, I. Shlosman, \& R.J. Weymann (San Francisco: ASP), p.52

Vanden Berk, D.E., et al. 2001, AJ, 122, 549

Véron-Cetty, M.-P., Véron, P., \& Tarenghi, M. 1983, A\&A, 119, 69

Véron-Cetty, M.-P. \& Véron, P. 2001, "A Catalogue of Quasars and Active Nuclei", 10th edition

Vestergaard, M. \& Wilkes, B.J. 2001, ApJS, 134, 1

Wampler, E.J., Gaskell, C.M., Burke, W.L., \& Baldwin, J.A. 1984, ApJ, 276, 403

Wandel, A. 1999a, ApJ, 527, 649

Wandel, A. 1999b, ApJ, 527, 657

Wanders, I., et al. 1997, ApJS, 113, 69

Wang, T.-G., Lu, Y.-J., \& Zhou, Y.-Y. 1998, ApJ, 493, 1

Weymann, R.J., Morris, S.L., Foltz, C.B., \& Hewett, P.C. 1991, ApJ, 373, 23

Weymann, R.J., Jannuzi, B.T., Lu, L., et al. 1998, ApJ, 506, 1

Wilkes, B.J., Tananbaum, H., Worrall, D.M., et al. 1994, ApJS, 92, 53

Wilkes, B.J., Kuraszkiewicz, J., Green, P.J., Mathur, S., \& McDowell, J.C. 1999, ApJ, 513, 76

Wills, B.J., Thompson, K.L., Han, M., et al. 1995, ApJ, 447, 139

Wills, B.J., Laor, A., Brotherton, M.S., et al. 1999, ApJ, 515, L53

Zamorani, G., Marano, B., Mignoli, M., Zitelli, V., \& Boyle, B.J. 1992, MNRAS, 256, 238

Zheng, W., Fang, L.-Z., \& Binette, L. 1992, ApJ, 392, 74

Zheng, W. \& Malkan, M.A. 1993, ApJ, 415, 517 
Zheng, W., Kriss, G.A., \& Davidsen, A.F. 1995, ApJ, 440, 606

Zheng, W., Kriss, G.A., Telfer, R.C., et al. 1997, ApJ, 475, 469 
Fig.1 - Redshift distribution of the AGN sample as a function of intrinsic luminosity at $\lambda=1450 \AA$. The open symbols represent radio-loud quasars and the filled symbols radioquiet quasars. The dashed horizontal (vertical) lines indicate the luminosity (redshift) ranges which were used to calculate composite spectra. At the left side (top) of the figure is the number of the individual spectra contributing to each composite spectrum. For the luminosity range $46.16 \leq \log \lambda L_{\lambda}(1450) \leq 47.16$ composite spectra were calculated for the redshift intervals marked by the vertical dashed lines.

Fig.2 - Composite spectrum of the entire AGN sample (top panel). The dashed line shows a power law fit to the continuum $(1200 \AA \leq \lambda \leq 5800 \AA)$ yielding $\alpha=-0.43\left(F_{\nu} \propto \nu^{\alpha}\right)$. The bottom panel displays the number of AGN contributing to each wavelength element.

Fig. 3 - (a) Normalized composite spectra are shown for redshift bins of $\Delta z=0.5$, starting at $z=0.5$ (cf., Fig. 1) and nearly constant luminosity $\left(46.16 \leq \log \lambda L_{\lambda}(1450) \leq\right.$ 47.16). The spectra were normalized by a power law continuum fit. The horizontal dashed lines indicate the continuum level for the individual normalized composite spectra which were vertically shifted for better display. The normalized continuum strength is shown for the spectrum at the bottom of the figure. This same scale applies to all other spectra.

(b) Same as Fig. 3a, but with an expanded vertical scale to display the dependence of the relative strength of weaker emission lines as a function of redshift. Strong emission lines with flat tops are truncated for easier display.

Fig. 4 - (a) Line equivalent widths, $W_{\lambda}$, vs. redshift for nearly constant intrinsic continuum luminosity $\lambda L_{\lambda}(1450 \AA)$.

(b) Same as Fig. 4a, but for additional emission lines.

Fig. 5 - (a) Normalized composite spectra are shown for the luminosity bins $\left(\Delta \log \lambda L_{\lambda}(1450 \AA)=\right.$ 0.5 dex) as displayed in Fig. 1 . The spectra were normalized with the corresponding power law continuum fit. The horizontal dashed lines indicate the continuum-level for the individual normalized composite spectra which were vertically shifted for better display. The normalized continuum strength is shown for the spectrum at the bottom of the figure and it applies for the other spectra too.

(b) Same as Fig. 5a, but with an expanded vertical scale to display the dependence of the relative strength of weaker emission lines as a function of luminosity. Strong emission lines with flat tops are truncated for easier display.

Fig. 6 - Same as Fig. 5a, but the normalized composite spectra are shown at larger wavelengths. 
Fig. 7 - (a) Line equivalent widths, $W_{\lambda}$, as a function of increasing continuum luminosity $\lambda L_{\lambda}(1450 \AA)$. We calculated linear fits to $W_{\lambda}(\mathrm{L})$ for the entire luminosity range (dashed line), as well as a luminosities $\log \lambda L_{\lambda}(1450 \AA) \geq 44$ (dashed-dotted line).

(b) Same as Fig. 7a, but for additional emission lines.

Fig. 8 - Difference spectra with respect to the composite spectrum $\log \lambda L_{\lambda}(1450 \AA)=$ $47.32 \mathrm{erg} \mathrm{s}^{-1}$ are displayed to illustrate that $W_{\lambda}(\mathrm{N} v \lambda 1240)$ is nearly constant, regardless how $W_{\lambda}(\mathrm{Nv})$ is measured, while the other emission lines display a prominant BEff. The dotted line indicates the location of $\mathrm{N} v \lambda 1240$.

Fig. 9 - The slope of the Baldwin Effect as displayed in Fig. 7a and 7b as a function of the ionization energy $\chi_{i o n}$ needed to create the specific ions. Different lines of the same ion (He II $\lambda 1640$, He II $\lambda 4686$ and O III] $\lambda 1663$, [O III] $\lambda 5007)$ show nearly identical slopes. The filled symbols represent the slopes based on the entire luminosity range, corresponding to the dashed lines in Figures 7a,b. The slopes of the Baldwin Effect for higher luminosities only $\left(\log \lambda L_{\lambda}(1450 \AA) \gtrsim 44\right)$ are plotted as open symbols. 


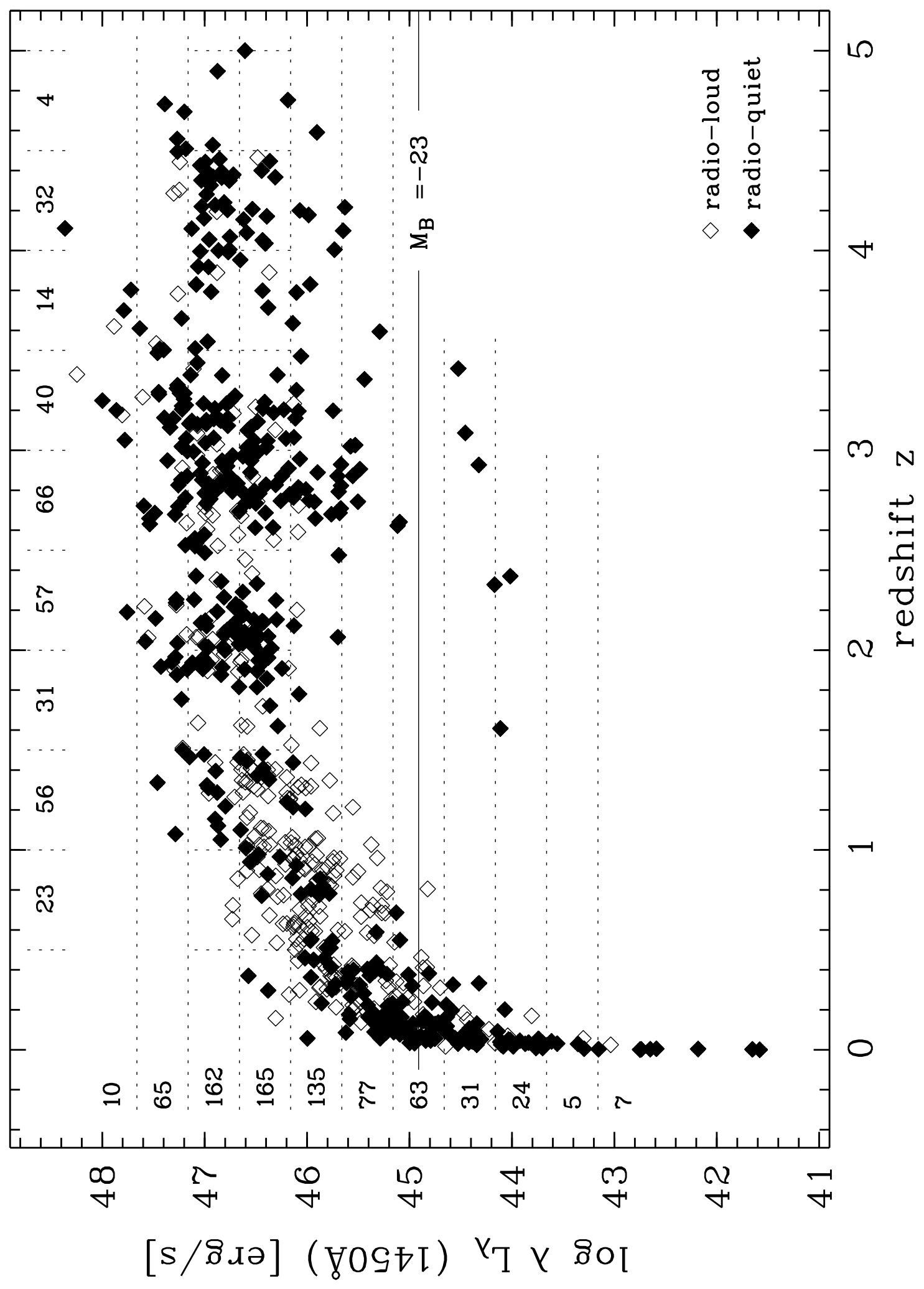




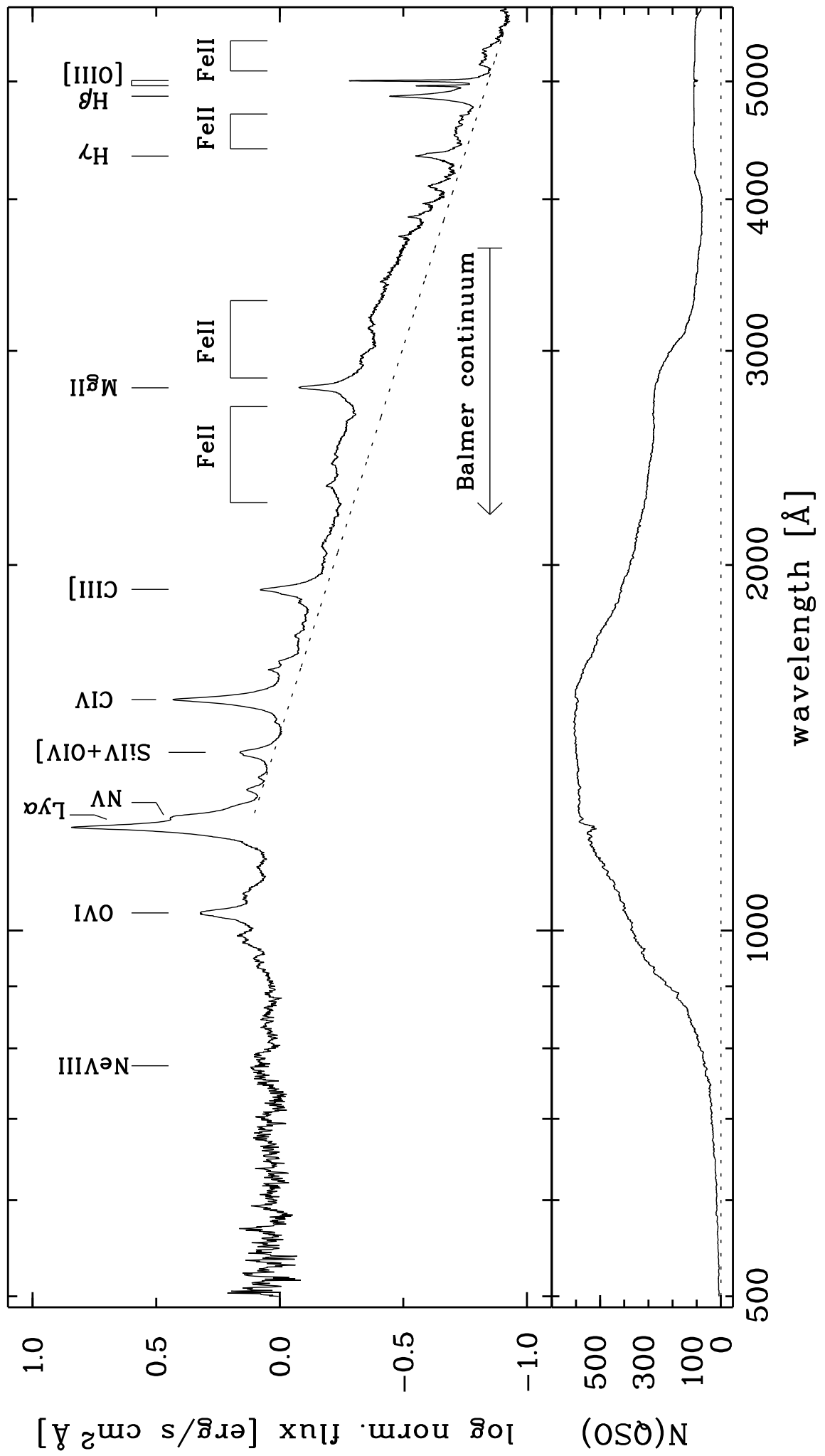



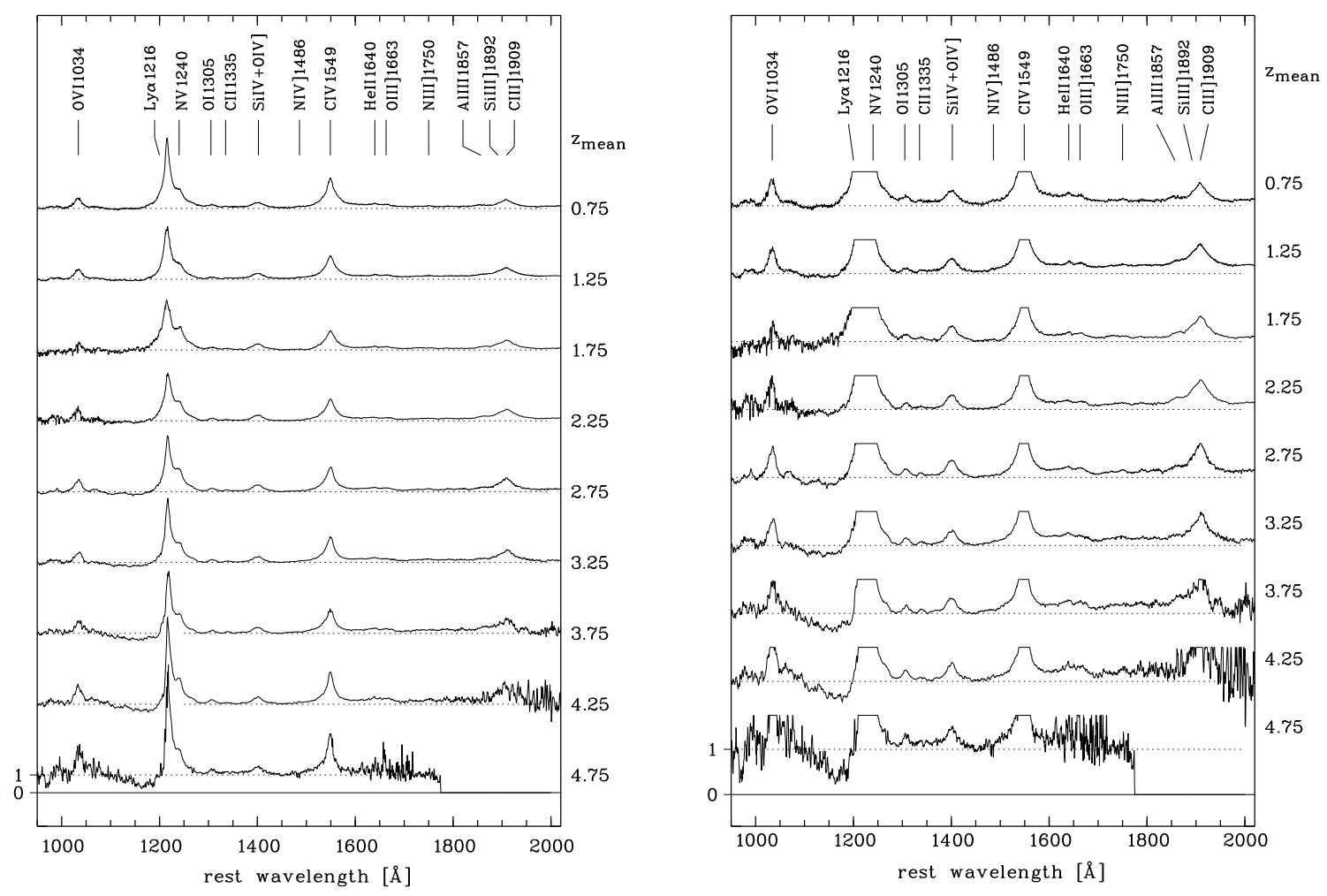

Fig. 3.- (a) Normalized composite spectra are shown for redshift bins of $\Delta z=0.5$, starting at $z=0.5$ (cf., Fig. 1) and nearly constant luminosity $\left(46.1 \leq \log \lambda L_{\lambda}(1450) \leq 47.1\right)$. The spectra were normalized with the corresponding power law continuum fit. The horizontal dashed lines indicate the continuum-level for the individual normalized composite spectra which were vertically shifted for better display. The normalized continuum strength is shown for the spectrum at the bottom of the figure and it applies for the other spectra, too. (b) Same as (a), but with an expanded vertical scale to display the dependence of the relative strength of weaker emission lines as a function of redshift. Strong emission lines with flat tops are truncated for easier display. 

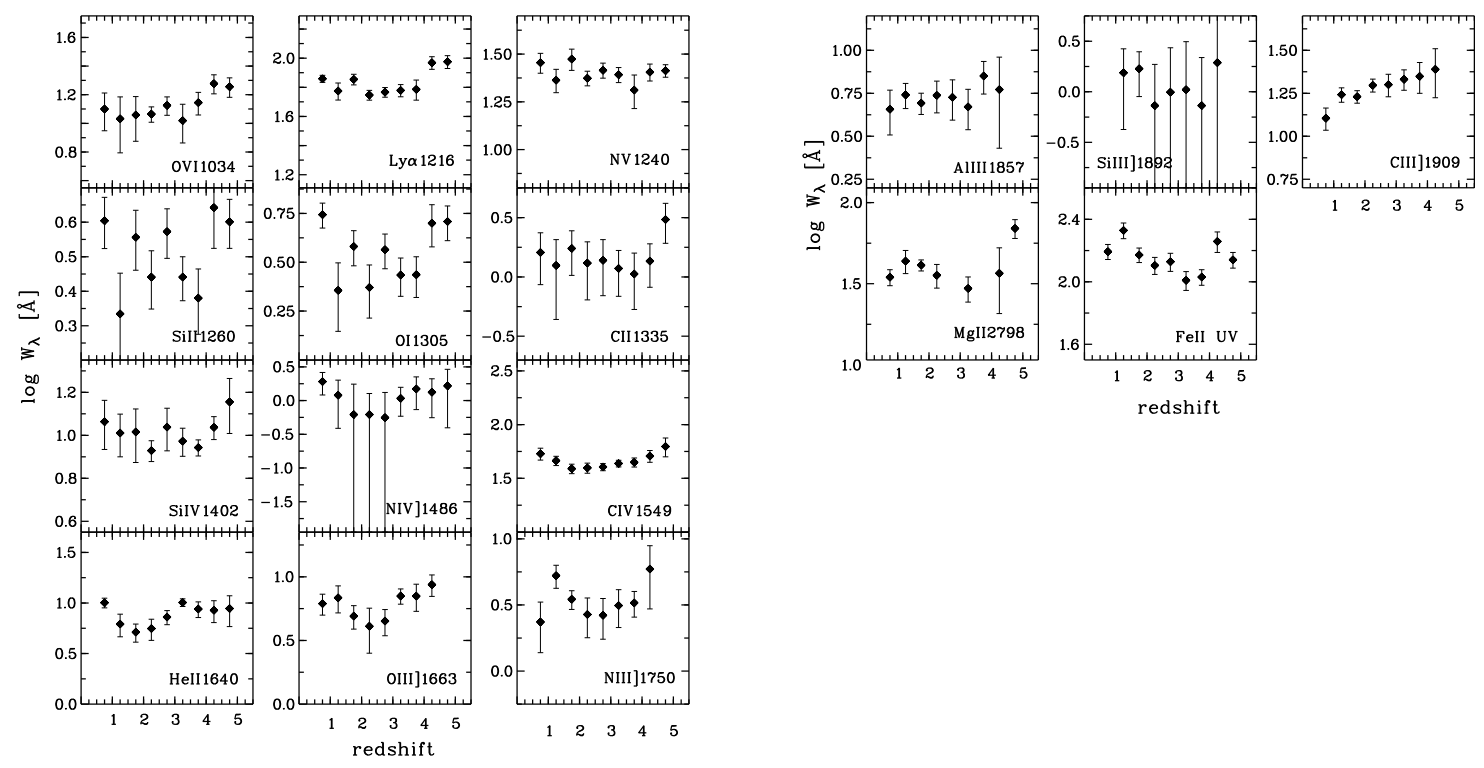

Fig. 4.- Line equivalent widths, $W_{\lambda}$, vs. redshift for nearly constant intrinsic continuum luminosity $\lambda L_{\lambda}(1450 \AA)$. 

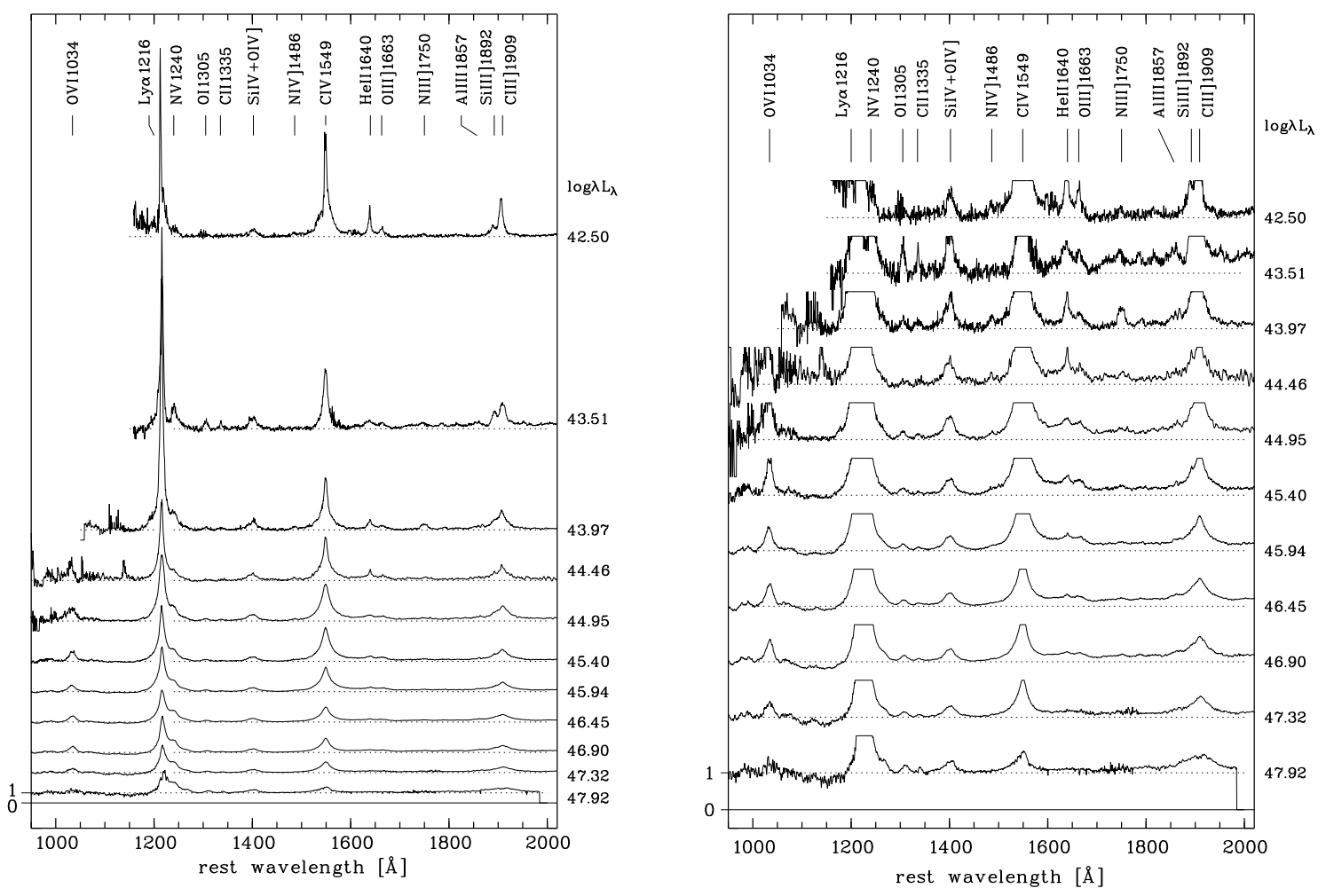

Fig. 5.- (a) Normalized composite spectra are shown for the luminosity bins $\left(\Delta \log \lambda L_{\lambda}(1450 \AA)=0.5\right.$ dex $)$ as displayed in Fig. 1. The spectra were normalized with the corresponding power law continuum fit. The horizontal dashed lines indicate the continuumlevel for the individual normalized composite spectra which were vertically shifted for better display. The normalized continuum strength is shown for the spectrum at the bottom of the figure and it applies for the other spectra, too. (b) Same as (a), but with an expanded vertical scale to display the dependence of the relative strength of weaker emission lines as a function of luminosity. Strong emission lines with flat tops are truncated for easier display. 


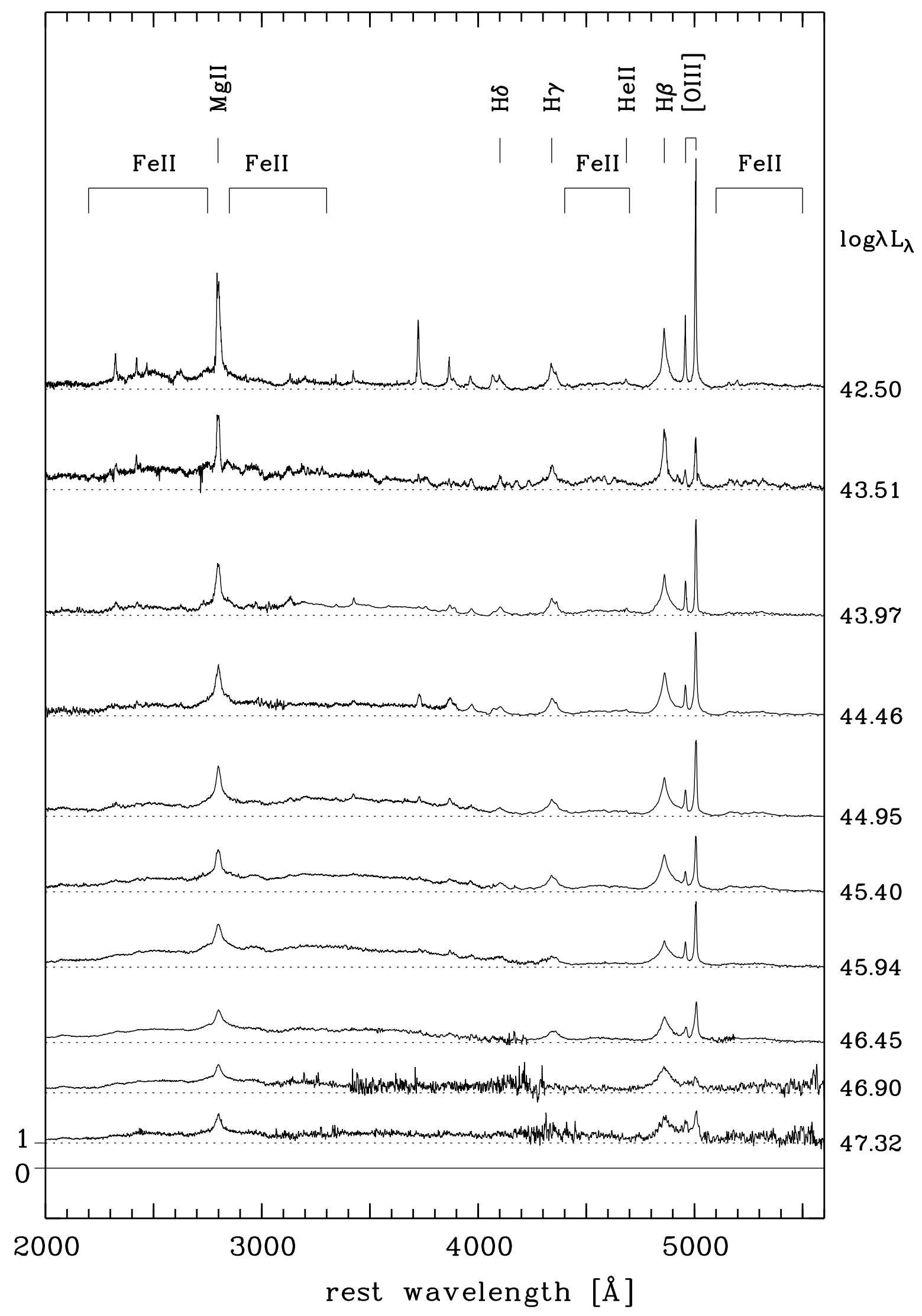



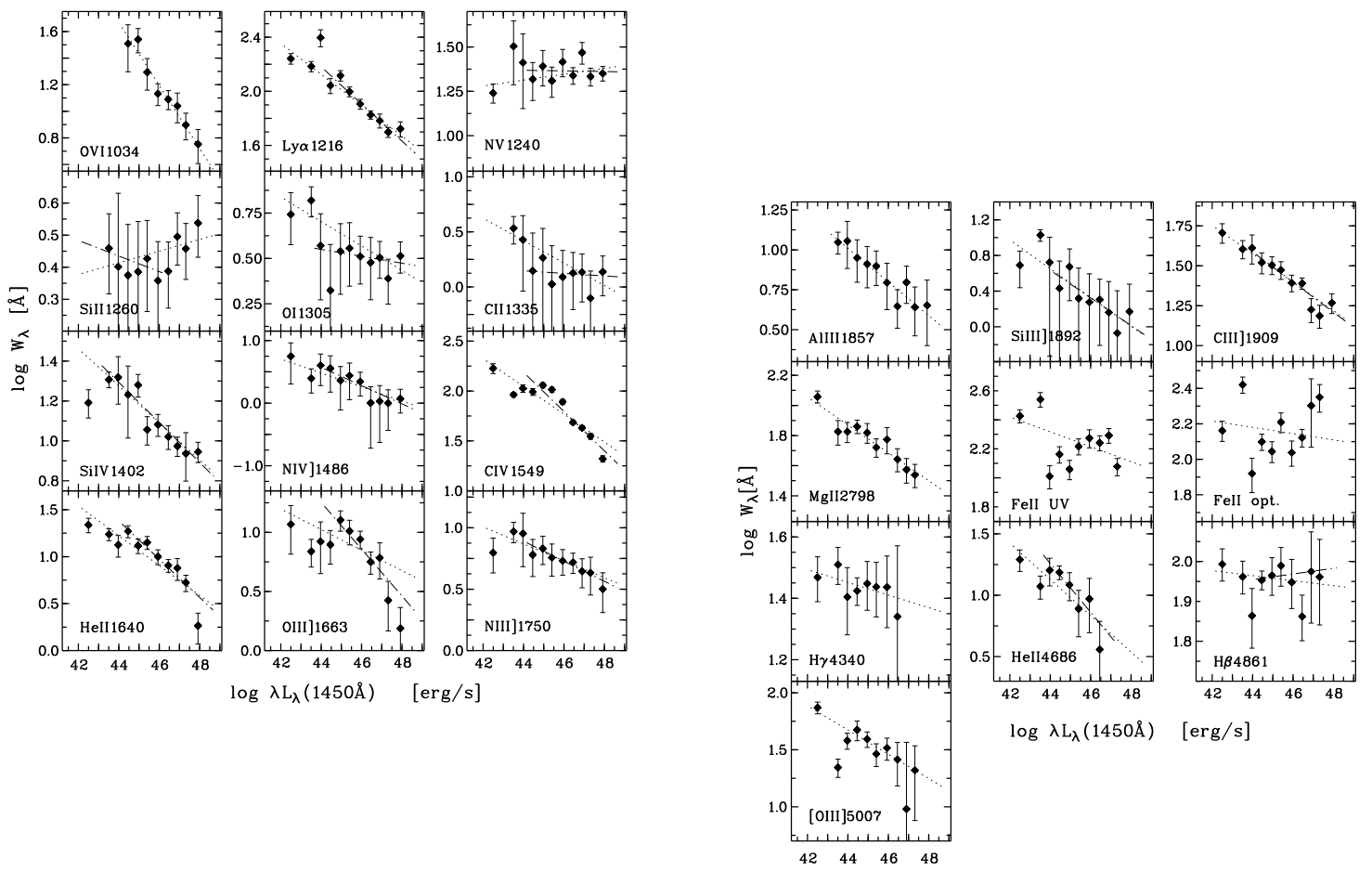

Fig. 7.- Line equivalent widths, $W_{\lambda}$, as a function of increasing continuum luminosity $\lambda L_{\lambda}(1450 \AA)$. We calculated linear fits to $W_{\lambda}(\mathrm{L})$ for the entire luminosity range (dashed line), as well as a luminosties $\log \lambda L_{\lambda}(1450 \AA) \geq 44$ (dashed-dotted line). 


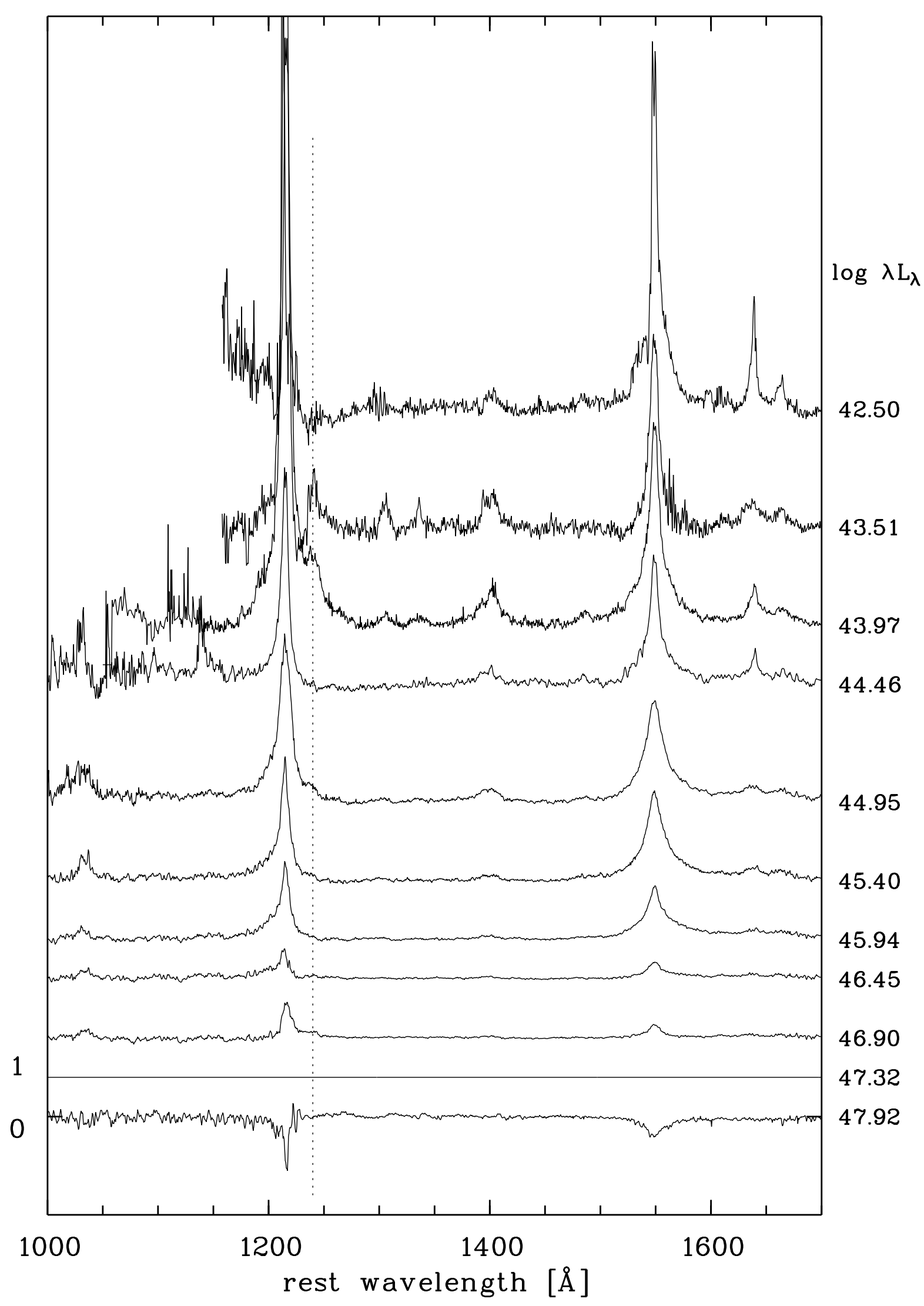




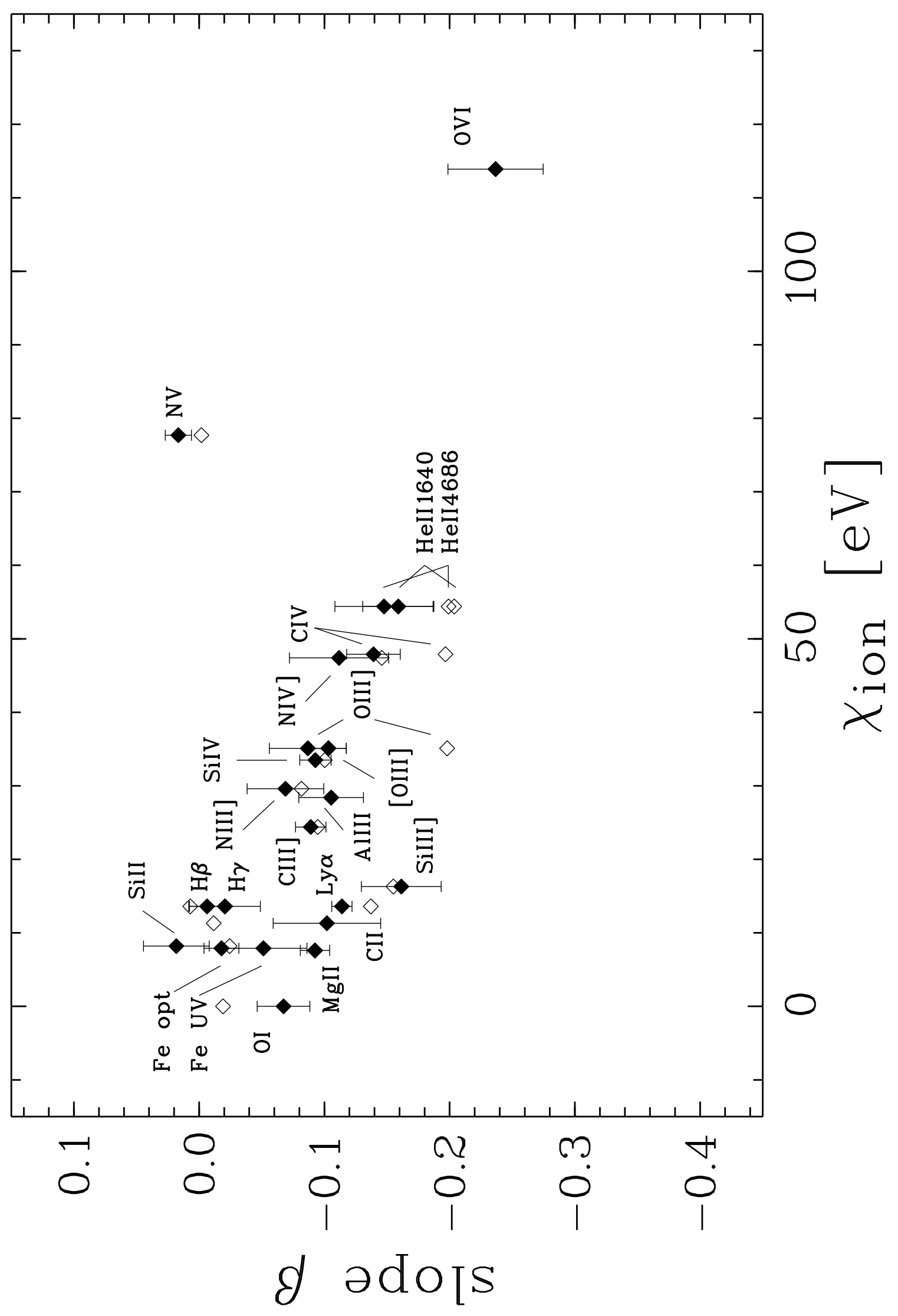


Table 1: Slope $\beta$ of the Baldwin Effect for the individual emission lines. A linear least square fit was calculated for the entire luminosity range and for $\log \lambda L_{\lambda}(1450 \AA) \gtrsim 44$; $\log W_{\lambda}=a+\beta \log \lambda L_{\lambda}(1450 \AA)$. In addition, for comparison theoretical predictions are given (Korista et al. 1998).

\begin{tabular}{|c|c|c|c|c|}
\hline line & $\begin{array}{l}\chi_{i o n} \\
{[\mathrm{eV}]}\end{array}$ & $\begin{array}{c}\text { entire } \lambda L_{\lambda}(1450 \AA) \text {-range } \\
\beta\end{array}$ & $\begin{array}{c}\log \lambda L_{\lambda}(1450 \AA) \\
\beta\end{array}$ & $\begin{array}{c}\text { Korista et al.(1998) } \\
\beta \\
\end{array}$ \\
\hline$\overline{O V I} \lambda 1034$ & 113.9 & $-0.24 \pm 0.04$ & $-0.24 \pm 0.04$ & -0.22 \\
\hline N v $\lambda 1240$ & 77.7 & $+0.01 \pm 0.01$ & $-0.00 \pm 0.02$ & -0.05 \\
\hline He II $\lambda 1640$ & 54.4 & $-0.16 \pm 0.03$ & $-0.20 \pm 0.04$ & -0.17 \\
\hline Не II $\lambda 4686$ & 54.4 & $-0.15 \pm 0.04$ & $-0.20 \pm 0.09$ & $\ldots$ \\
\hline C IV $\lambda 1549$ & 47.9 & $-0.14 \pm 0.02$ & $-0.20 \pm 0.03$ & -0.20 \\
\hline $\mathrm{N}$ IV] $\lambda 1486$ & 47.4 & $-0.11 \pm 0.04$ & $-0.14 \pm 0.08$ & -0.01 \\
\hline О III] $\lambda 1663$ & 35.1 & $-0.09 \pm 0.03$ & $-0.20 \pm 0.05$ & -0.12 \\
\hline [O III $] \lambda 5007$ & 35.1 & $-0.10 \pm 0.02$ & $\ldots$ & $\ldots$ \\
\hline Si IV $\lambda 1402$ & 33.5 & $-0.09 \pm 0.01$ & $-0.10 \pm 0.02$ & -0.13 \\
\hline N III] $] \lambda 1750$ & 29.6 & $-0.07 \pm 0.03$ & $-0.08 \pm 0.04$ & +0.09 \\
\hline $\mathrm{Al}$ III $\lambda 1857$ & 28.4 & $-0.11 \pm 0.03$ & $-0.11 \pm 0.03$ & -0.04 \\
\hline C III] $\lambda 1909$ & 24.4 & $-0.09 \pm 0.01$ & $-0.09 \pm 0.02$ & -0.08 \\
\hline Si III] $\lambda 1892$ & 16.3 & $-0.16 \pm 0.03$ & $-0.16 \pm 0.12$ & -0.06 \\
\hline Ly $\alpha \lambda 1216$ & 13.6 & $-0.11 \pm 0.01$ & $-0.14 \pm 0.02$ & -0.10 \\
\hline $\mathrm{H} \gamma \lambda 4340$ & 13.6 & $-0.02 \pm 0.03$ & $-0.02 \pm 0.03$ & $\ldots$ \\
\hline $\mathrm{H} \beta \lambda 4861$ & 13.6 & $-0.01 \pm 0.01$ & $+0.01 \pm 0.03$ & $\ldots$ \\
\hline C II $\lambda 1335$ & 11.3 & $-0.10 \pm 0.04$ & $-0.01 \pm 0.09$ & +0.00 \\
\hline Si II $\lambda 1260$ & 8.2 & $+0.02 \pm 0.03$ & $-0.01 \pm 0.09$ & $\ldots$ \\
\hline Fe IIUV & 7.9 & $-0.05 \pm 0.03$ & $\ldots$ & $\ldots$ \\
\hline Fe IIopt & 7.9 & $-0.02 \pm 0.03$ & $\ldots$ & $\ldots$ \\
\hline $\operatorname{Mg} \operatorname{II} \lambda 2798$ & 7.6 & $-0.09 \pm 0.01$ & $-0.09 \pm 0.01$ & -0.09 \\
\hline O I $\lambda 1305$ & 0.0 & $-0.07 \pm 0.02$ & $-0.02 \pm 0.04$ & -0.05 \\
\hline
\end{tabular}

